\title{
A review of the genus Norellisoma Wahlgren, 1917 (Diptera: Scathophagidae) of Russia
}

\section{Обзор видов рода Norellisoma Wahlgren, 1917 (Diptera: Scathophagidae) фауны России}

\author{
A.L. Ozerov ${ }^{1}$, M.G. Krivosheina ${ }^{2}$ \\ А. $\Lambda$. Озеров $^{1}$, М.Г. Кривошеина ${ }^{2}$
}

\author{
'Zoological Museum, Moscow Lomonosov State University, Bol'shaya Nikitskaya 2, Moscow 125009, Russia. \\ E-mail: ozerov2455@rambler.ru \\ 'Зоологический музей, Московский государственный университет им. М.В. Ломоносова, Большая Никитская ул., 2, Москва \\ 125009 , Россия \\ ${ }^{2}$ A.N. Severtsov Institute of Ecology and Evolution, Russian Academy of Sciences, 119071 Moscow, Russia. \\ E-mail: dipteramarina@rambler.ru \\ ${ }^{2}$ Институт проблем экологии и эволюции им. А.Н.Северцова РАН, Ленинский проспект, 33, Москва 119071, Россия.
}

KEY WORDS: Diptera, Scathophagidae, Norellisoma, Russia, review, new synonyms, new records.

КЛЮЧЕВЫЕ СЛОВА: Diptera, Scathophagidae, Norellisoma, Россия, обзор, новые синонимы, новые находки.

ABSTRACT. Flies of the genus Norellisoma Wahlgren, 1917 of Russia, which includes 12 species, are reviewed. Generic and species descriptions and key for determination of species are given, and data on distributions are summarized. One new synonym is proposed: Norellisoma lituratum (Meigen, 1826) = N. altaicum (Ozerov, 2008), syn.n. New records of the following species are given: Norellisoma caucasicum (Ozerov, 1993) (Azerbaijan, Kazakhstan); N. lesgiae (Becker, 1894), N. montanopratense (Ozerov, 1993) (Abkhazia); N. spinimanum (Fallén, 1819) (Kazakhstan).

PЕЗЮМЕ. Дан обзор двукрылых рода Norellisoma Wahlgren, 1917 фауны России, включающего 12 видов. Приведены диагноз рода, описания видов и ключ для определения видов рода Norellisoma России, а также суммированы данные по распространению видов рода на территории России. Установлен один новый синоним: Norellisoma lituratum (Meigen, 1826) $=N$. altaicum (Ozerov, 2008), syn.n. Приведены новые данные о распространении следующих видов: Norellisoma caucasicum (Ozerov, $1993)$ (Азербайджан, Казахстан); N. lesgiae (Becker, 1894), N. montanopratense (Ozerov, 1993) (Абхазия); N. spinimanum (Fallén, 1819) (Казахстан).

\section{Introduction}

Norellisoma Wahlgren, 1917 is not large genus within the family Scathophagidae and consists of 28 species distributed in the Northern Hemisphere (1 Hol- arctic, 27 Palaearctic) [Vockeroth, 1965; Šifner, 2008; Ozerov, 2008, 2009, 2010a; Ozerov, 2013].

The fauna of Russia includes 12 species [Becker, 1894; Gorodkov, 1970, 1986; Šifner, 2008; Ozerov, 2008, 2009, 2010a, b; Ovchinnikov, 2013; Ozerov, Krivosheina, 2014; Bagachanova et al., 2016], but no general work has been fulfilled.

Adults of some species of Norellisoma are often collected at locations where Rumex spp. (Polygonaceae) grow, usually on the leaves of these plants. I collected adults of $N$. armipes in a meadow of confluent of the Ruza river with Urtica dioica (Urticaceae) and Campanula latifolia (Campanulaceae) [Ozerov, 2010a]. Adults were on leaves of C. latifolia in the second half of May till about the second half of June before plants formed buds or flowers.

Larvae of $N$. spinimanum (Fallén) mine stems of Rumex [Ferrar, 1987]. Description of immature stages of $N$. spinimanum (Fallén) was given by Disney [1976].

\section{Material and methods}

The specimens examined for this study are deposited in the Zoological Museum, Moscow State University, Russia (ZMUM) and Zoological Institute of Russian Academy of Sciences, St. Petersburg, Russia (ZISP). We also studied some material from the Institute of Systematics and Ecology of Animals, Russian Academy of Sciences, Siberian Branch, Novosibirsk, Russia (ISEA) and Oxford University Museum, Oxford, England (UMO).

How to cite this article: Ozerov A.L., Krivosheina M.G. 2020. A review of the genus Norellisoma Wahlgren, 1917 (Diptera: Scathophagidae) of Russia // Russian Entomol. J. Vol.29. No.1. P.103-120. doi: 10.15298/ rusentj.29.1.15 


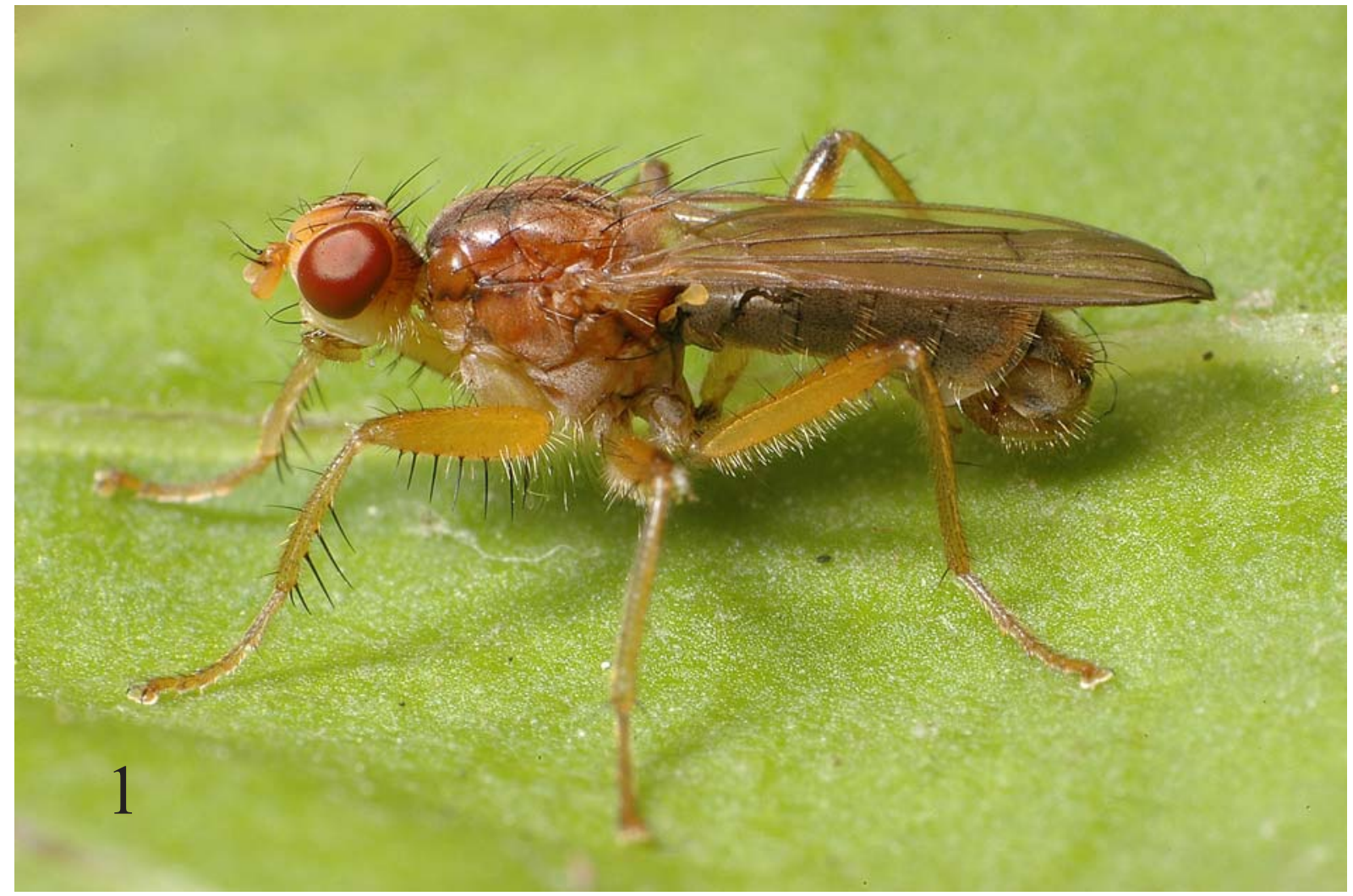

Fig. 1. Male of Norellisoma spinimanum (Fallén). After Ozerov, 2010a, fig. 1.

Рис. 1. Самец Norellisoma spinimanum (Fallŭn). По Ozerov, 2010a, fig. 1.

The majority of original Russian geographical names are given in transliteration, but names of some large geographical regions (e.g. Altai (=Altai Krai + Altai Republic), Krasnoyarsk Krai, Primorsky Krai, Sakha Republic (=Yakutia), Zabaykalsky Krai) follow these indicated in Google Earth software.

The terminology used in the generic and species descriptions follows McAlpine [1981], Cumming \& Wood [2009], and Stuckenberg [1999].

\section{Taxonomic part}

\section{Norellisoma Wahlgren, 1917}

Norellisoma Hendel, 1910: 308. Nomen nudum.

Norellisoma Wahlgren, 1917: 148 [as Norellisoma Hendel, 1910]. Gender: neuter. Type-species: Cordylura spinimana Fallén, 1819 , by designation of Vockeroth, 1965.

Norelliosoma, error.

Norellisoma species are small or medium-sized flies (3.5-8.7 mm long). Colour varies from yellow to black.

Head. Frontal vitta yellow, rarely blackish in upper part, matt; fronto-orbital plate yellow completely or blackish in upper part, greyish dusted; ocellar triangle black. Face, parafacial and gena yellowish, whitish dusted. Postcranium usually blackish in upper half and yellow in lower part or yellow almost completely, greyish dusted, usually covered with black setulae in upper third and pale hairs in lower part. Setae: 2 orbitals, 2-4 frontals, 1 ocellar, 1 postocellar (small, divergent), 1 inner vertical, 1 outer vertical; one pair of vibrissae present. Antenna from yellow to blackish. Postpedicel rounded apically, approximately 2-2.5 times as long as wide. Arista yellowish or blackish, basally thickened, pubescent. Clypeus and proboscis yellowish or brownish. Palpus elongate and slender, yellow, without long apical seta.

Thorax from yellow to black, grey dusted. Scutum with the following paired setae: $(1+2)+(2+3)$ dorsocentrals, scapular pair present or absent, intra-alars absent, (0-1)+2 supra-alars, 0-1 postpronotal, 1-2 notopleurals, 1-2 postalars. Proepisternum with hairs in central part, with $0-1$ seta near lower margin. Proepimeron with $0-1$ seta and several hairs. Anepisternum covered with hairs in posterior half, with 13 setae near posterior margin. Katepisternum covered with hairs in posterior half and with one strong seta in upper posterior corner. Anepimeron bare. Postmetacoxal bridge absent. Scutellum greyish dusted, with a pair of strong discal scutellar setae (Fig. 3).

Legs yellow in ground color, sometimes mid and hind femora blackish dorsally. Fore femur with rows of long posteroventral and of short anteroventral setae. Fore tibia with 4-7 strong posteroventral and 5-8 strong anteroventral setae (Fig. 2), also with 1-2 dorsal/anterodorsal, 1 posterodorsal and 1 preapical dorsal setae. Mid femur with 3-6 setae anteriorly in api- 


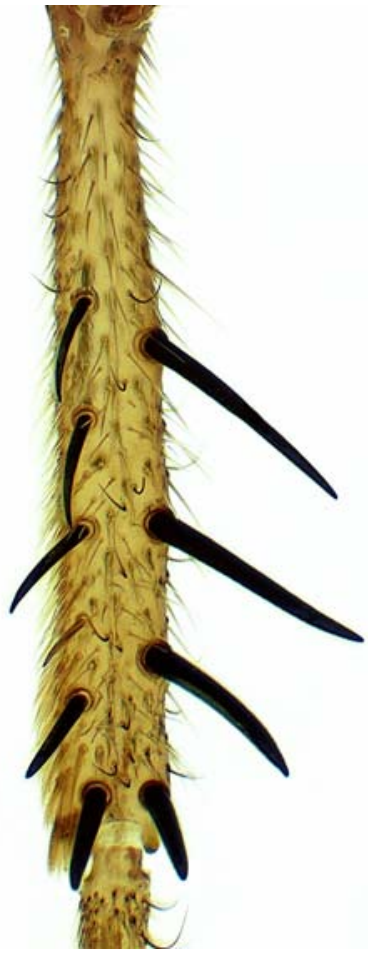

2

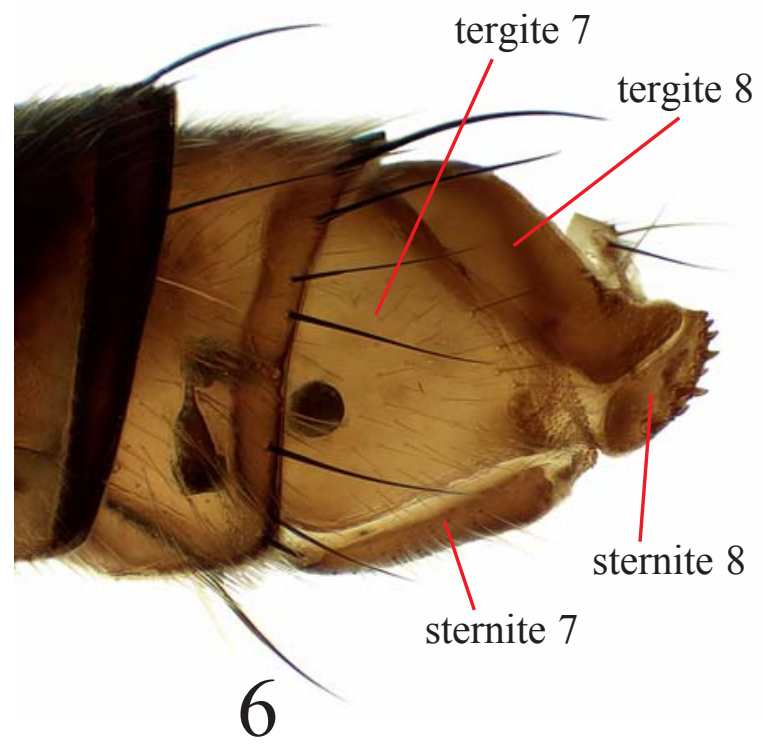

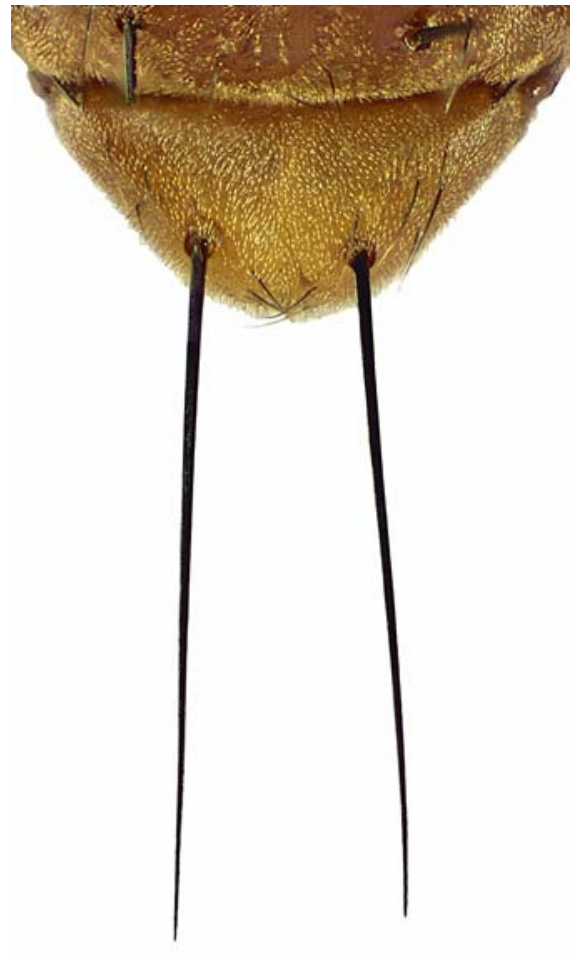

3

\section{西}

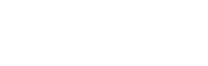

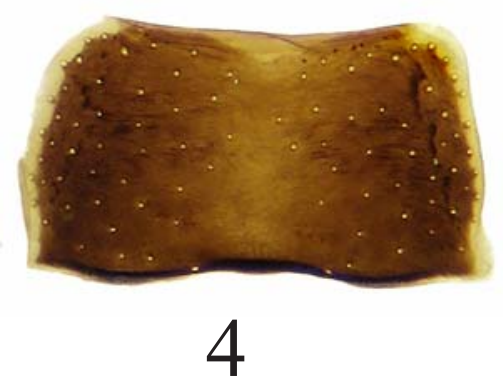

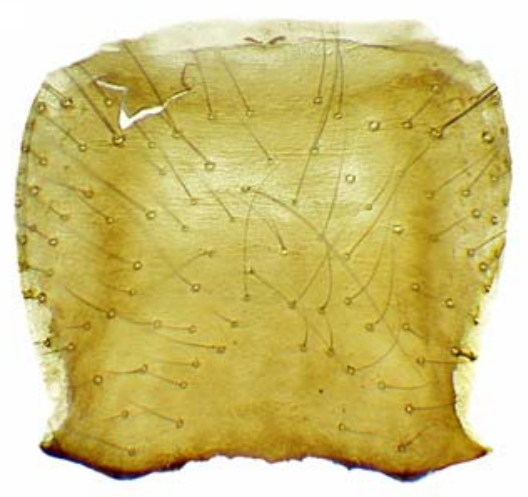

5

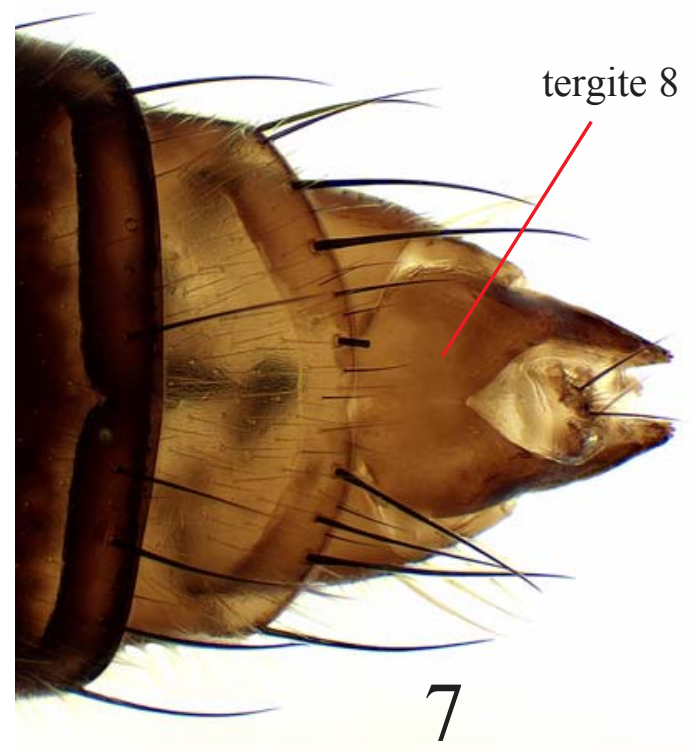

Figs 2-7. Norellisoma spp.: 2 - right fore tibia of $N$. spinimanum (Fallén), ventral view; 3 - scutellum of $N$. spinimanum (Fallén), dorsally; 4 - male sternite 4 of $N$. caucasicum (Ozerov); 5 - male sternite 4 of $N$. spinimanum (Fallén); 6 - end of female abdomen of $N$. spinimanum (Fallén), lateral view; 7 - same, dorsal view. 2, 3 - after Ozerov \& Krivosheina, 2011, figs 2, 4; 5 - after Ozerov, 2010a, fig. 6.

Рис. 2-7. Norellisoma spp.: 2 - голень правой передней ноги N. spinimanum (Fallén), снизу; 3 - щиток N. spinimanum (Fallén), сверху; 4 - стернит 4 самца N. caucasicum (Ozerov); 5 - стернит 4 самца N. spinimanum (Fallén); 6 - конец брюшка самки $N$. spinimanum (Fallén), сбоку; 7 - то же, сверху. 2, 3 - по Ozerov \& Krivosheina, 2011, figs 2, 4; 5 - по Ozerov, 2010a, fig. 6. 
cal part, with 1 preapical posterior seta and 2-3 antero-ventral setae in apical third, in males of some species additionally with hairs ventrally. Mid tibia with 1-2 anteroventral, 1-2 anterodorsal, 1-2 posterodorsal, 1-3 posteroventral setae and a ring of apicals, in male of some species additionally with long hairs ventrally. Hind femur with a row of anterodorsal setae, with 1-4 anteroventral setae in apical quarter. Hind tibia with 1-3 anterodorsal, 2-3 posterodorsal, 0-1 anteroventral in apical quarter, 1 preapical dorsal and 1 apical antero-ventral setae.

Wing tinged with whitish or brownish, veins brownish; vein $\mathrm{R}_{1}$ bare dorsally (rarely (some specimens of $N$. lituratum) with 1-2 setulae dorsally near apex). Calypters, margins of calypters, and halteres yellowish or brownish.

Abdomen cylindrical, black, greyish dusted. Female segments 7-8 subshining or shining, flattened laterally. Male sternite 4 rectangular, as wide as long (Fig. 5) or wider than long (Fig. 4). Sternite 5 with narrow or wide triangular lateral lobes, in some species with short median projections (Figs 11, 16). Male terminalia (Figs 20-54): epandrium with symmetrical surstyli having the appearance of ticks; surstyli often broaded apically; cerci fused apically or medially forming cercal plate. Female ovipositor short, compressed laterally, with proctiger shifted dorsally (Figs 6,7); tergite 8 in form of ring not closed in distal part (Figs 6,7), sternite 8 covered with spines (Fig. 6).

\section{Norellisoma armipes Meigen, 1826} Figs 8, 20-22.

armipes Meigen, 1826: 234 (Cordylura). Type-locality: locality not stated.

flavicauda Meigen, 1826: 235 (Cordylura). Type-locality: not given.

flava von Roser, 1840: 59 (Cordylura). Type-locality: not given (?Württemberg).

roserii Rondani, 1866: 17 (Norellia). Type-locality: “Specimen germanicum..." (Germany).

MATERIAL. Moscow Oblast: Ignatkovo env. $\left(55.9395^{\circ} \mathrm{N}\right.$ 35.6225 ${ }^{\circ}$ ), 10 and 15.VI.2011, 23.VI.2012, A.L. Ozerov (6 $\sigma^{7} \sigma^{7}, 1$, ZMUM); Ivanovskoe env. $\left(55.939^{\circ} \mathrm{N} 35.621^{\circ} \mathrm{E}\right), 12$.VI.2006, A.L. Oze$\operatorname{rov}\left(1 \mathrm{O}^{\prime}, 1\right.$ ㅇ, ZMUM); Naro-Fominsk $\left(55.372^{\circ} \mathrm{N} 36.757^{\circ} \mathrm{E}\right), 14 . \mathrm{V} .2010$ D. Gavryushin (1 $\sigma^{\prime}$, ZMUM); Anikeevka $\left(55.827^{\circ} \mathrm{N} 37.214^{\circ} \mathrm{E}\right)$, 23.VIII.1983, Gorodkov (3 $\sigma^{7} \sigma^{\top}, 1$ ㅇ, ZISP).

ADDITIONAL MATERIAL. Ukraina: Truskavets $\left(49.278^{\circ} \mathrm{N}\right.$ 23.510 $0^{\circ}$ E), 16.VII.1980, Narchuk (1 $\sigma^{7}$, ZISP).

DESCRIPTION. Male, female. Medium-sized flies (7.2-8.3 mm long).

Head. Frontal vitta and fronto-orbital plate yellow. Postcranium usually blackish in upper half and yellow in lower part, rarely yellow almost completely. 3-4 frontal setae present. Antenna yellow, usually darkened around base of arista. Postpedicel approximately 2 times as long as wide. Arista blackish, moderate haired: the length of hairs more than the width of basal diameter of arista.

Thorax usually black, grey dusted; sometimes scutum and pleural sclerites partly yellow. Scutum with the following paired setae: $2+3$ dorsocentrals, a pair of scapular setae (usually small), 1+2 supraalars, 1 postpronotal, 2 notopleural (posterior seta small), 2 postalars. Anepisternum with 1 seta near posterior margin. Scutellum yellow or brown, delicately greyish dusted.

Legs entirely yellow, but usually mid and hind femora blackish dorsally. Fore femur with 7-8 long posteroventral and 6-9 short anteroventral setae. Fore tibia with 4 strong posteroventral and 5-6 strong anteroventral setae. Male mid femur with more or less dense hairs ventrally. Mid tibia with 1 anteroventral, 1 anterodorsal, 1-2 posterodorsal, 2-3 posteroventral and a ring of apical setae, in male additionally with long hairs ventrally. Hind tibia with 2-3 anterodorsal, 2-3 posterodorsal, 1 anteroventral, 1 preapical dorsal and 1 apical anteroventral setae.

Wing tinged with brownish. Calypters, margins of calypters, and halteres yellowish.

Abdomen black, greyish dusted. Female segments 7-8 shining. Male sternite 4 approximatelly as wide as long. Male sternite 5 with broad and short lobes covered with spinules inside (Fig. 8). Epandrium, cercal plate and surstyli as in Figs 20-22.

DISTRIBUTION. Russia: Moscow Oblast. - Europe (France, Germany, Ukraine) [Gorodkov, 1986; Ozerov, 2009].

Norellisoma caucasicum (Ozerov, 1993) Figs 4, 9, 23-25.

caucasica Ozerov, 1993: 68 (Norellia). Type-locality: $10 \mathrm{~km}$ SE of Alagir, Bakhty Lapparyrag ridge, $1600 \mathrm{~m}$ (North Ossetia Alania, Russia).

MATERIAL. Altai: Aktash env, $\left(50.32^{\circ} \mathrm{N} 87.73^{\circ} \mathrm{E}\right), 2600 \mathrm{~m}$, 6.VII.2016, N. Vikhrev (1 $0^{7}$, ZMUM); Seminsky pass, $\left(51.0^{\circ} \mathrm{N}\right.$ $\left.85.5^{\circ} \mathrm{E}\right), 1650 \mathrm{~m}, 27-30$. VI.2016, N. Vikhrev (3 OO+, ZMUM); Karachay-Cherkessia: Arkhyz $\left(43.562^{\circ} \mathrm{N} 41.282^{\circ} \mathrm{E}\right)$, 14.VII.1939, D'yakonov, (1 $\left.\bigcirc^{\top}, \mathrm{ZISP}\right)$; Teberdinskiy Reserve $\left(43.443^{\circ} \mathrm{N} 41.737^{\circ} \mathrm{E}\right)$, 3-6.V.2000, K. Tomkovich (1 $\sigma^{7}$, ZMUM); Krasnodar Krai: Teberdinskiy Reserve (ca. $43.443^{\circ} \mathrm{N} 41.738^{\circ} \mathrm{E}$ ), $2700 \mathrm{~m}, 25-29 . \mathrm{VI} .1968$, Gorodkov (5 $\sigma^{7} \sigma^{7}, 3$ 우, ZISP); Lagonaki $\left(44.00^{\circ} \mathrm{N} 39.09^{\circ} \mathrm{E}\right), 1700$ m, 11.VI.2012, 5-7.V.2013, N. Vikhrev (1 $\sigma^{7}, 1$ q, ZMUM); Lagonaki $\left(44.0^{\circ} \mathrm{N} 39.9^{\circ} \mathrm{E}\right), 1500-1900 \mathrm{~m}, 5-9 . V I .2015$, N. Vikhrev (3 $\sigma^{7} \sigma^{7}, 1$ ㅇ, ZMUM); Estosadok env., Psekhako Mt. $\left(43.691^{\circ} \mathrm{N}\right.$ 40.366 ${ }^{\circ} \mathrm{E}$ ), 14-18.VI.2008, K. Tomkovich (5 $\sigma^{7} \sigma^{7}, 1$ ㅇ, ZMUM); North Ossetia - Alania: Buron env. (43.793 N 43.922 $\left.{ }^{\circ} \mathrm{E}\right)$, 7.VI.1989, 4 and 13.VII.1990, A.L. Ozerov (3 $\sigma^{\top} \sigma^{\top}$, ZMUM); Bakhty-Laparyrag ridge $\left(42.938^{\circ} \mathrm{N} 44.287^{\circ} \mathrm{E}\right), 1770 \mathrm{~m}, 29 . \mathrm{VII} .1988,23-31 . V .1989$ and 28-30.VI.1990, A.L. Ozerov (14 $\sigma^{\top} \sigma^{\top}, 5$ 우, ZMUM); same place, 28-29.VI.1990, A. Shatalkin (3 $\sigma^{7} \sigma^{7}$, ZMUM).

ADDITIONAL MATERIAL. Azerbaijan: Damir-Aparanchay River $\left(41.066^{\circ} \mathrm{N} 47.933^{\circ} \mathrm{E}\right), 1600-1700 \mathrm{~m}, 15$. VII.1994, M. Savitskiy (2 $\bigcirc^{\top} \sigma^{7}$, ZMUM); Kazakhstan: Katon-Karagay NP., Verkhnee Zimovye terrain env. $\left(49.083^{\circ} \mathrm{N} 86.033^{\circ} \mathrm{E}\right), 26-27 . \mathrm{VII} .2010$, O. Kosterin (1 $\sigma^{7}, 1$, ZMUM).

DESCRIPTION. Male, female. Small or mediumsized flies (3.8-5.6 mm long).

Head. Frontal vitta and fronto-orbital plate each yellow completely or blackish in upper half. Postcranium blackish (in male rarely yellow along lower margin). 2 frontal setae present. Antenna yellow completely in male, but in female postpedicel usually blackish on outside surface. Postpedicel approximately 2 times as long as wide. Arista blackish, short haired: the length 


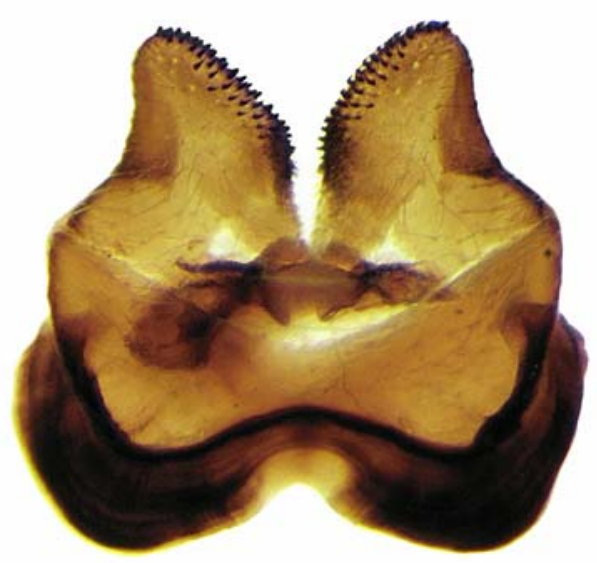

8
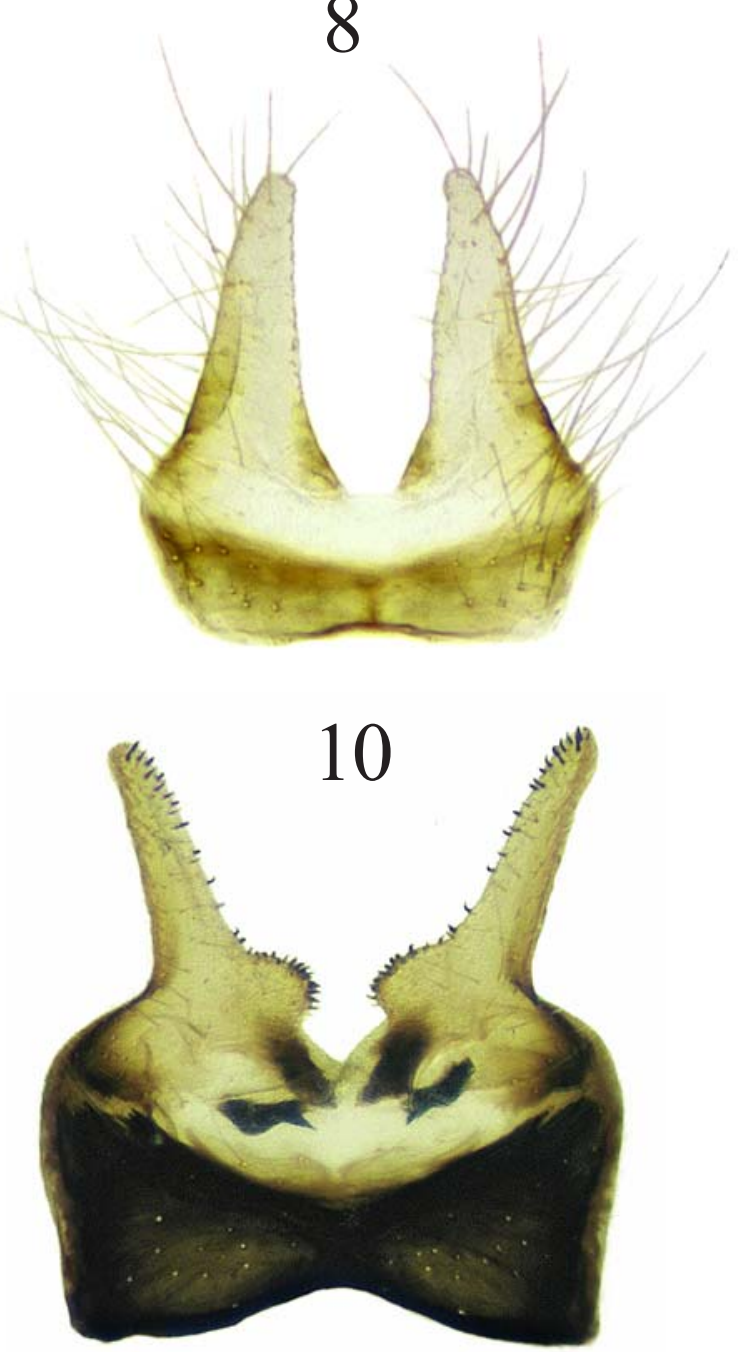

12

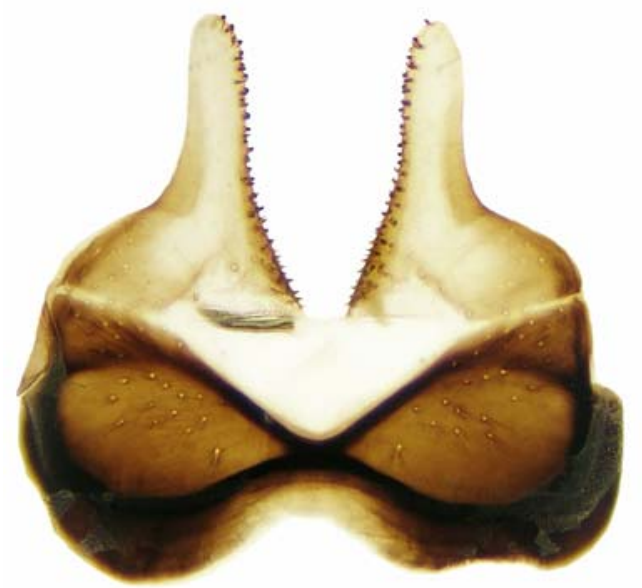

9

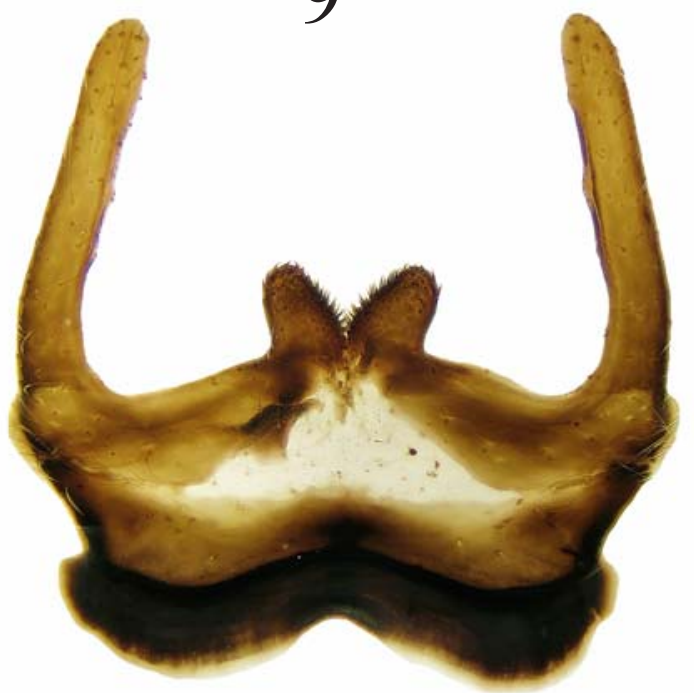

11

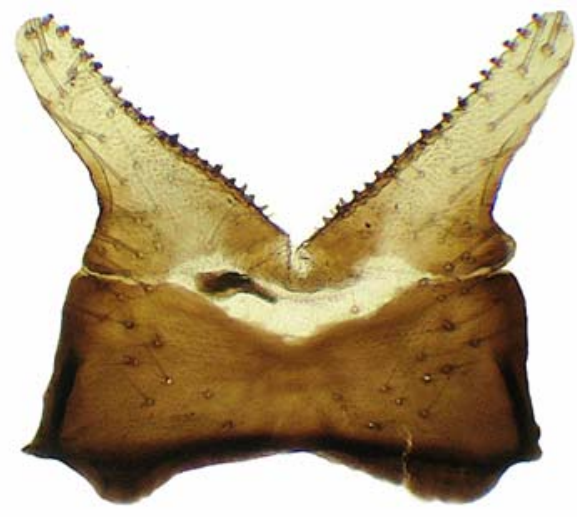

13

Figs 8-13. Male sternite 5 of Norellisoma spp.: $8-N$. armipes (Meigen); $9-N$. caucasicum (Ozerov); $10-N$. flavostriatum Ozerov; $11-N$. insulare (Ozerov); $12-N$. lesgiae (Becker); $13-N$. lituratum (Meigen). 8, 12, 13 - after Ozerov, 2010a, figs 10, 26, 29; 9 - after Ozerov, 2010b, fig. 15; 10 - after Ozerov, 2009, fig. 15.

Рис. 8-13. Стернит 5 самца Norellisoma spp.: $8-$ N. armipes (Meigen); $9-N$. caucasicum (Ozerov); $10-N$. flavostriatum Ozerov; $11-N$. insulare (Ozerov); $12-N$. lesgiae (Becker); $13-N$. lituratum (Meigen). 8, 12, 13 - по Ozerov, 2010a, figs 10, 26, 29; 9 - по Ozerov, 2010b, fig. 15; 10 - по Ozerov, 2009, fig. 15. 

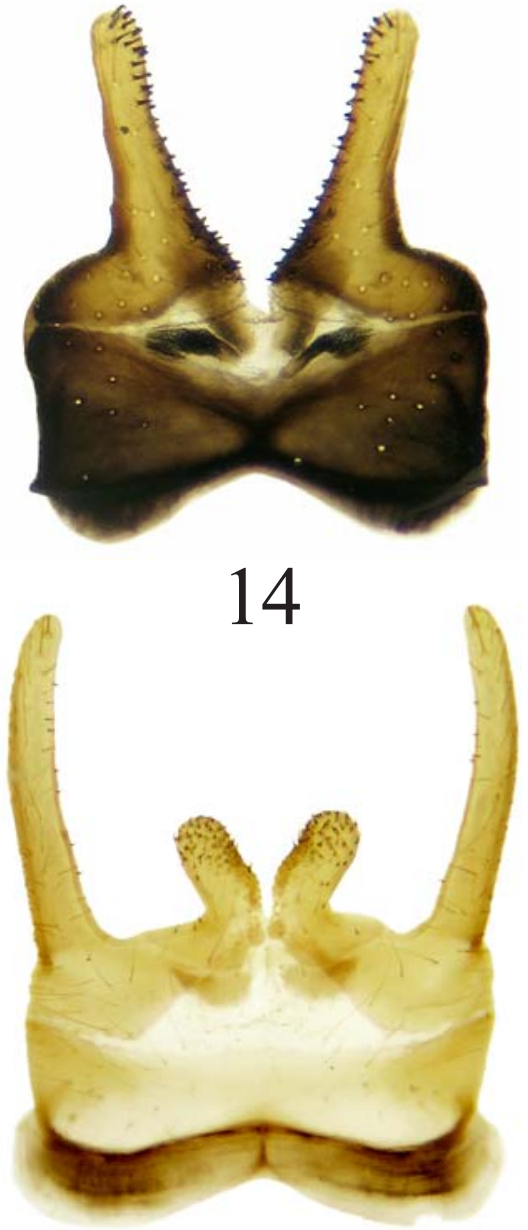

16

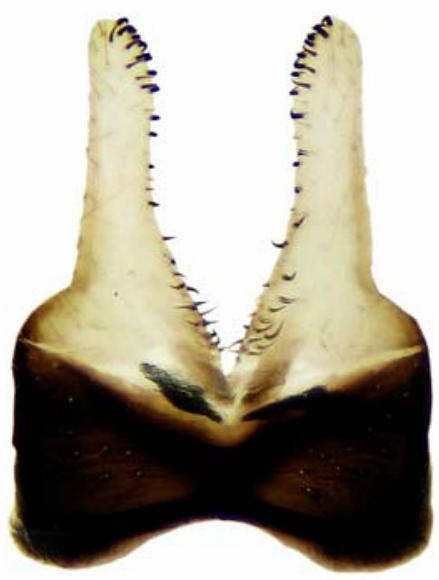

18
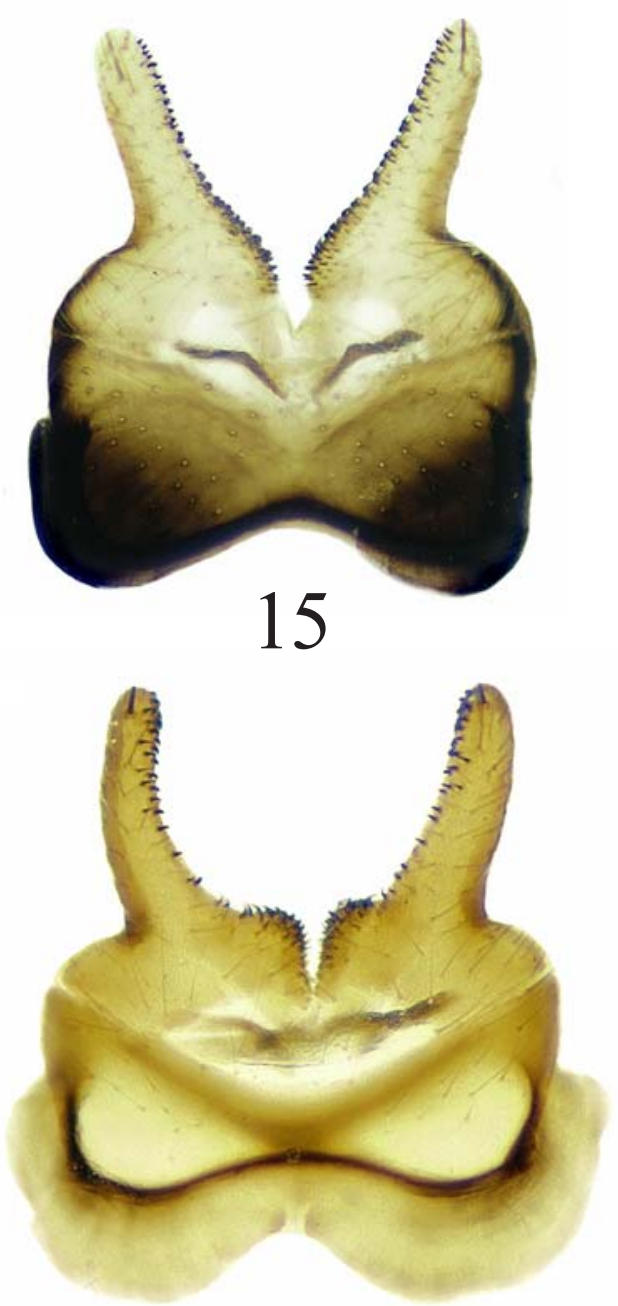

17

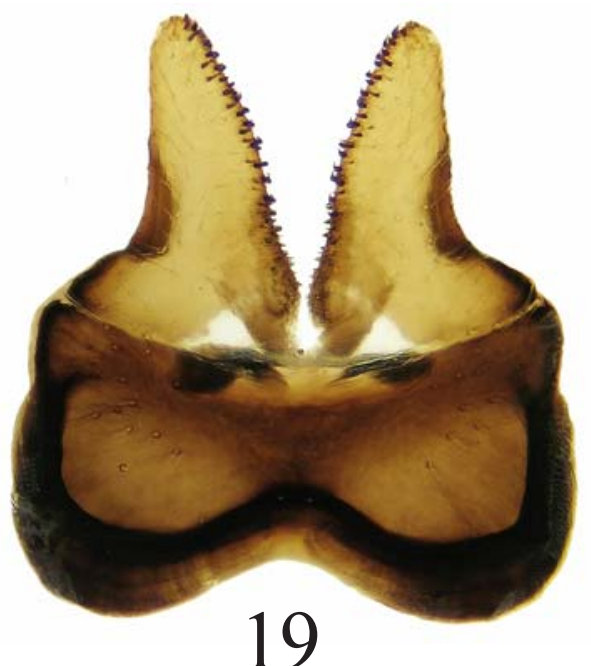

Figs 14-19. Male sternite 5 of Norellisoma spp.: $14-N$. montanopratense (Ozerov); $15-N$. oreinum Ozerov; $16-N$. orientale (Ozerov); $17-N$. spinimanum (Fallén); $18-N$. tomkovichi Ozerov; $19-N$. yolduense (Ozerov). 15, 17 - after Ozerov, 2010a, figs 5, 22; 18 - after Ozerov, 2010b, fig. 14.

Рис. 14-19. Стернит 5 самца Norellisoma spp.: $14-N$. montanopratense (Ozerov); $15-N$. oreinum Ozerov; $16-N$. orientale (Ozerov); $17-$ N. spinimanum (Fallén); $18-N$. tomkovichi Ozerov; $19-N$. yolduense (Ozerov). 15, 17 — по Ozerov, 2010a, figs 5, 22; 18 — по Ozerov, 2010b, fig. 14. 
of hairs less than the width of basal diameter of arista.

Thorax and scutellum black, grey dusted. Scutum with the following paired setae: $2+2$ dorsocentrals (anterior presutural seta usually small), $1+2$ supra-alars, 1 postpronotal, 2 notopleurals, 2 postalars. Anepisternum with 1-2 setae near posterior margin.

Legs yellow in ground color, but often (usually in female) darkened dorsally. Fore femur with 9-11 long posteroventral, 7-8 short anteroventral, 5-7 dorsal/posterodorsal setae. Fore tibia with 5 strong posteroventral and 5-7 strong anteroventral setae. Mid tibia with 2 anteroventral, 1-2 anterodorsal, 1-2 posterodorsal, 2-3 posteroventral and a ring of apical setae. Hind tibia with 2-3 anterodorsal, 2-3 posterodorsal, 1 anteroventral, 1 preapical dorsal and 1 apical anteroventral setae.

Wing tinged with brownish. Calypters, margins of calypters, and halteres yellowish.

Abdomen black, greyish dusted. Female segments 7-8 shining. Male sternite 4 wider than long (Fig. 4). Male sternite 5 with lobes broad basally and narrowed apically (Fig. 9). Epandrium, cercal plate and surstyli as in Figs 23-25.

DISTRIBUTION. Russia: Altai, Karachay-Cherkessia, Krasnodar Krai, North Ossetia - Alania. - Azerbaijan (first record), Kazakhstan (first record).

\section{Norellisoma flavostriatum Ozerov, 2009} Figs 10, 26, 27.

flavostriatum Ozerov, 2009: 423 (Norellisoma). Type-locality: Estosadok env., Psekhako Mt. (4341'28"N, 40²2'E) (Krasnodar Krai, Russia).

NOTE. This species is known only from the typelocality [Ozerov, 2009].

DESCRIPTION. Male. Length of body $4.8 \mathrm{~mm}$.

Head. Frontal vitta and fronto-orbital plate yellow. Postcranium blackish in upper part, excluding yellow median occipital sclerite, and whitish in lower half. 2 frontal setae present. Antenna yellow. Postpedicel approximately 2 times as long as wide. Arista yellowish, moderate haired: the length of hairs more than the width of basal diameter of arista.

Thorax delicately greyish dusted. Scutum black with yellow stripe between dorsocentral setae. Lateral thoracic sclerites yellow, only anepimeron, katatergite, anatergite and mediotergite black. Scutum with the following paired setae: $1+1$ dorsocentrals, $0+2$ supraalars, postpronotals absent, 1 notopleural (anterior), 1 postalar. Anepisternum with 1 seta near posterior margin. Scutellum yellow, grey dusted.

Legs entirely yellow. Fore femur with 10-11 long and thin posteroventral, 9-12 short anteroventral, 4-5 dorsal/posterodorsal setae. Fore tibia with 7 strong posteroventral and 7-8 anteroventral setae. Mid tibia with 1 anteroventral, 1 anterodorsal, 2 posterodorsal setae, with a row of moderate long hairs posteroventrally, and a ring of apical setae. Hind tibia with 1-2 anterodorsal, 2 posterodorsal, 1 preapical dorsal and 1 apical anteroventral setae.
Wing tinged with whitish, darkened near apexes of veins $R_{2+3}$ and $M_{1}$. Calypters, margins of calypters, and halteres yellowish.

Abdomen black, greyish dusted. Male sternite 5 with conical lobes covered with long hairs (Fig. 10). Epandrium, cercal plate and surstyli as in Figs 26, 27.

Female unknown.

DISTRIBUTION. Russia: Krasnodar Krai.

Norellisoma insulare (Ozerov, 1993)

Figs 11, 28-30.

insularis Ozerov, 1993: 71 (Norellia). Type-locality: Kunashir I., environs of the Mendeleev volcano (Sakhalin Oblast, Russia).

NOTE. This species was recorded in Russia from Far East by Ozerov \& Krivosheina [2014].

MATERIAL. Sakhalin Oblast: Kunashir I., cape Alekhinsky $\left(43.918^{\circ} \mathrm{N} 145.526^{\circ} \mathrm{E}\right), 19-21 . I X .2009$, I. Melnik (1 $\sigma^{7}, 1$ \% , ZMUM); Kunashir I., State Nature reserve Kurilskiy, caldera of the Golovnin volcano $\left(43.84^{\circ} \mathrm{N} 145.50^{\circ} \mathrm{E}\right), 3-5 . V I I .2014$, I.A. Gomyranov (1 ㅇ, ZMUM); Kunashir I., State Nature reserve Kurilskiy, cordon Andreevskiy $\left(43.5^{\circ} \mathrm{N} 145.3^{\circ} \mathrm{E}\right), 6-8 . V I I .2014$, I.A. Gomyranov (1 $\sigma^{7}$, ZMUM); Kunashir I., State Nature reserve Kurilskiy, cordon Filatovskiy $\left(44.1^{\circ} \mathrm{N} 146.0^{\circ} \mathrm{E}\right), 18-19 . \mathrm{VII} .2014$, I.A. Gomyranov (1 o, ZMUM); Kunashir I., Tretyakovo $\left(43.991^{\circ} \mathrm{N} 145.655^{\circ} \mathrm{E}\right), 13-$ 22.IX.2009, I. Melnik (1 +, ZMUM); Kunashir I., Alekhino $\left(43.95^{\circ} \mathrm{N}\right.$ $\left.145.592^{\circ} \mathrm{E}\right)$, 5.VI.1968, Narchuk (1 $\sigma^{7}$, ZISP); same place, 5-6.VI.1968, V. Richter (4 $\sigma^{7} \sigma^{\top}, 7$ ㅇ, ZISP); Kunashir I., Mendeleevo (43.954 $\mathrm{N}$ 145.681 $\left.{ }^{\circ} \mathrm{E}\right), 2-9 . V I .1968, \mathrm{~V}$. Richter (4 $\sigma^{\top} \sigma^{7}, 11$ OO , ZISP); same place, 3.VI. and 28.VI.1968, Narchuk (4 우, ZISP); same place, 25.VI.1973, Kerzhner (1 9 , ZISP); Kunashir I., environs of the Mendeleev volcano $\left(43.961^{\circ} \mathrm{N} 145.729^{\circ} \mathrm{E}\right)$, 4.VII.1985, S. Churkin (2 OO+, ZMUM); Kunashir I., Sernovodsk (43.912 $\left.{ }^{\circ} \mathrm{N} 145.641^{\circ} \mathrm{E}\right), 8 . \mathrm{VI} .1968$, V. Richter (6 $\bigcirc^{7} \sigma^{7}, 13$ 우, ZISP); Kunashir I., Tret'yakovo $\left(43.989^{\circ} \mathrm{N}\right.$ $\left.145.644^{\circ} \mathrm{E}\right)$, 4.VIII.1973, Kasparyan (1 $\sigma^{7}$, ZISP); Kunashir I., Yuzhno-Kuril'sk $\left(44.029^{\circ} \mathrm{N} 145.859^{\circ} \mathrm{E}\right), 16 . V I .1968$, V. Richter, Narchuk ( $\sigma^{7}, 1$, ZISP); Kunashir I., Ivanovskyi cape $\left(43.82^{\circ} \mathrm{N} 145.40^{\circ} \mathrm{E}\right)$, 23-29.V.2013, Yu. Sundukov (3 $\sigma^{7} \sigma^{7}, 1$ \%, ZMUM); Kunashir I., Filatovka River $\left(44.18^{\circ} \mathrm{N} 146.02^{\circ} \mathrm{E}\right), 25-27 . V I .2013$, Yu. Sundukov ( $3 \bigcirc^{7} \bigcirc^{7}$, ZMUM); Kunashir I., the mouth of the stream Bystryi $\left(43.78^{\circ} \mathrm{N}\right.$

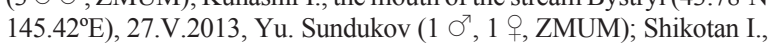
Tserkovnaya Bay $\left(43.7^{\circ} \mathrm{N} 146.7^{\circ} \mathrm{E}\right), 23-31 . V .2012$, Yu. Sundukov (7

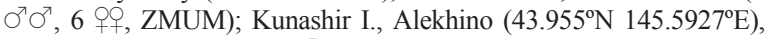
13.VI.1973, Kerzhner (1 $\sigma^{7}$, ZISP); Sakhalin I., Pravda $\left(46.941^{\circ} \mathrm{N}\right.$ $\left.142.008^{\circ} \mathrm{E}\right), 25 . V .1973$, Kerzhner (2 $\sigma^{\top} \sigma^{\top}, 2$ 우, ZISP); Sakhalin I., Novoaleksandrovsk $\left(47.056^{\circ} \mathrm{N} 142.730^{\circ} \mathrm{E}\right), 24$ and 29.V.1968, Narchuk (3 OO․ ZISP); same place, 23.V.1973, Kerzhner (1 ㅇ, ZISP); Sakhalin I., $30 \mathrm{~km} \mathrm{~W}$ of Yuzhno-Sakhalinsk $\left(46.96^{\circ} \mathrm{N} 142.244^{\circ} \mathrm{E}\right)$, 28.VI.1968, Narchuk (1 ๑), 1 , ZISP); Sakhalin I., Chekhov Mt. $\left(48.870^{\circ} \mathrm{N} 144.60^{\circ} \mathrm{E}\right), 24$.VIII.1973, Logvinovskiy (1 $\sigma^{7}$, ZISP); Sakhalin I., Yuzhno-Sakhalinsk (46.954 $\left.{ }^{\circ} \mathrm{N} 142.736^{\circ} \mathrm{E}\right)$, 7.VI.1954, Violovich (5 ○7 $\sigma^{7}, 5$ 우, ZISP); Sakhalin I., Tymovo env. $\left(51.128^{\circ} \mathrm{N}\right.$ 142.675 ${ }^{\circ}$ E), 1.VI.1979, A. Veselkin (1 $\sigma^{7}$, ZMUM).

DESCRIPTION. Male, female. Medium-sized flies (6.1-8.6 mm long).

Head. Frontal vitta and fronto-orbital plate yellow. Postcranium usually blackish in upper half and yellow in lower part, rarely yellow almost completely. 3-4 frontal setae present. Antenna yellow. Postpedicel approximately 2 times as long as wide. Arista blackish, moderately haired: the length of hairs more than the width of basal diameter of arista.

Thorax from yellow to blackish, delicately grey dusted. Scutum often yellow with two blackish stripes along dorsocentral lines or blackish completely between dorsocentral lines. Scutum with the following 
paired setae: $2+3$ dorsocentrals, a pair of scapular setae (usually small), 1+2 supra-alars, 1 postpronotal (rarely 2 setae), 2 notopleurals, 2 postalars. Anepisternum with 2-3 setae near posterior margin. Scutellum from yellow or blackish, delicately greyish dusted.

Legs entirely yellow, but usually mid and hind femora blackish dorsally near apex. Male femora and tibiae with hairs longer than in female. Fore femur with 8-9 long posteroventral and 8-11 short anteroventral setae. Fore tibia with 4 strong posteroventral and 5 strong anteroventral setae. Mid tibia with 1-2 anterodorsal, 1-2 posterodorsal and a ring of apical setae in both sexes, in male additionally with long pale hairs ventrally, in female with 1 anteroventral and 2-3 posteroventral setae. Hind tibia with 3-4 anterodorsal, 2-3 poste-rodorsal, 1 anteroventral, 1 preapical dorsal and 1 apical anteroventral setae.

Wing tinged with brownish. Calypters, margins of calypters, and halteres brownish.

Abdomen black, greyish dusted. Female segments 7-8 shining. Male sternite 4 wider than long. Male sternite 5 with narrow stick-like lateral lobes and two short median projections; length of median projection less than the distantion between median projection and lateral lobe (Fig. 11). Epandrium, cercal plate and surstyli as in Figs 28-30.

DISTRIBUTION. Russia: Sakhalin Oblast. — Japan [Hironaga, Suwa, 2005].

\section{Norellisoma lesgiae (Becker, 1894)} Figs 12, 31-33.

lesgiae Becker, 1894: 129 (Norellia). Type-locality: "Lesgia" [=Dagestan] (Russia).

NOTE. Noted by Gorodkov [1986] for European part of Russia from Caucasus, and from Georgia (without indicating specific locality).

MATERIAL. Karachay-Cherkessia: Dombay, valley of the Alibek River $\left(43.291^{\circ} \mathrm{N} 41.594^{\circ} \mathrm{E}\right), 2500 \mathrm{~m}, 1$. VII.1982, Narchuk (6 $0^{7} \mathrm{O}^{7}, 3$ 우, ZISP); Klukhor River (ca. $\left.43.250^{\circ} \mathrm{N} 41.834^{\circ} \mathrm{E}\right), 2200$ m, 25.VII.1905, Kalishevs[?kiy], (1 +, ZISP); Krasnodar Krai: Lagonaki $\left(44.009^{\circ} \mathrm{N} 39.09^{\circ} \mathrm{E}\right), 1700 \mathrm{~m}, 23-30$.VI.2011, 11.VI.2012, and 5-7.V.2013, N. Vikhrev (10 $0^{7} \sigma^{7}, 3$ 우, ZMUM); Lagonaki (44.0 $\left.0^{\circ} \mathrm{N} 39.9^{\circ} \mathrm{E}\right), 1500-1900 \mathrm{~m}, 5-9 . V I .2015$, N. Vikhrev (5 $\sigma^{7} \sigma^{7}$, 1 o, ZMUM); same place $\left(44.10^{\circ} \mathrm{N} 40.02^{\circ} \mathrm{E}, 1450 \mathrm{~m} ; 44.05^{\circ} \mathrm{N}\right.$ $\left.40.01^{\circ} \mathrm{E}, 1830 \mathrm{~m} ; 44.09^{\circ} \mathrm{N} 40.01^{\circ} \mathrm{E}, 1725 \mathrm{~m}\right), 15-28 . \mathrm{VI} .2009, \mathrm{~K}$ Tomkovich (5 $\sigma^{7} \sigma^{7}$, ZMUM); Krasnaya Polyana $\left(43.682^{\circ} \mathrm{N}\right.$ $\left.40.20^{\circ} \mathrm{E}\right), 24$.VI.1909, A. Yakovleva (1 9 , ZISP); Psebay $\left(44.114^{\circ} \mathrm{N}\right.$ $40.794^{\circ}$ E) near Shishi, 27.V.1911, Volnukhin (1 $0^{7}, 5$ 우, ZISP); Estosadok env., Psekhako Mt. (43.691 $\left.{ }^{\circ} \mathrm{N} 40.366^{\circ} \mathrm{E}\right), 14-18 . V I .2008$, K. Tomkovich ( $70^{7} \sigma^{7}, 1$,, ZMUM); Teberdinskiy Reserve, Badukskie Lakes $\left(43.376^{\circ} \mathrm{N} 41.660^{\circ} \mathrm{E}\right), 12 . \mathrm{VI} .1968$, Negrobov (1, , ZISP); Teberdinskiy Reserve $\left(43.252^{\circ} \mathrm{N} 41.829^{\circ} \mathrm{E}\right), 8$.VI.1964, 29.VIII.1965, Gorodkov (2 $0^{7} O^{7}$, ZISP); Teberdinskiy Reserve $\left(43.443^{\circ} \mathrm{N} 41.738^{\circ} \mathrm{E}\right), 20$. VI.-9.VII.1968, Gorodkov (63 O $^{7} \mathrm{O}^{7}, 40$ o+o, ZISP); North Ossetia - Alania: Buron env. $\left(43.793^{\circ} \mathrm{N}\right.$ 43.922 ${ }^{\circ}$ E), 17.VII.1988, 6.VI.-14.VII.1990, A.L. Ozerov (4 $0^{7} 0^{7}$, 9 우, ZMUM); same place, 3.VII.1990, A. Shatalkin (1 $\mathrm{O}^{7}$, ZMUM); Bakhty-Laparyrag ridge $\left(42.938^{\circ} \mathrm{N} 44.287^{\circ} \mathrm{E}\right), 1770 \mathrm{~m}, 23 . \mathrm{V}$.17.VI.1989, A.L. Ozerov (6 O $0^{7}, 7$ 우, ZMUM).

ADDITIONAL MATERIAL. Abkhazia: Bagri-Yashta ridge $\left(43.440^{\circ} \mathrm{N} 40.708^{\circ} \mathrm{E}\right), 2000 \mathrm{~m}, 2 . \mathrm{VII} .2009$, A. Gusakov (1 क ZMUM).

DESCRIPTION. Male, female. Medium-sized flies (4.8-8.2 mm long).
Head. Frontal vitta yellow; fronto-orbital plate yellow in lower part and blackish in upper half or third. Postcranium black in upper part and yellow in lower 1/ 3. 2 frontal setae present. Antenna yellow, but sometimes postpedicel in female partly blackish on outside surface. Postpedicel approximately 2 times as long as wide. Arista black, short haired: the length of hairs less than the width of basal diameter of arista.

Thorax and scutellum black, densely grey dusted. Scutum with the following paired setae: $2+2$ dorsocentrals, 1+2 supra-alars, 1 postpronotal, 1 or 2 notopleurals (posterior usually small or absent), 2 postalars. Anepisternum with 1 setae near posterior margin.

Legs yellow, rarely fore and mid femora darkened dorsally in some females. Male femora and tibiae with hairs longer than in female. Fore femur with 7-10 long posteroventral and 6-9 short anteroventral setae. Fore tibia with 4 strong posteroventral and 5-6 strong anteroventral setae. Mid tibia with 1-2 anteroventral, 1 anterodorsal, 1-2 posterodorsal and a ring of apical setae in both sexes, in male additionally with long pale hairs ventrally, in female with $0-1$ apical and 2-3 posteroventral setae. Hind femur with a row of black anterodorsal setae, with 1-3 anteroventral setae in apical quarter and $0-1$ apical dorsal seta. Hind tibia with 2-3 anterodorsal, 2 posterodorsal, 1 preapical dorsal and 1 apical antero-ventral setae.

Wing tinged with brownish. Calypters, margins of calypters, and halteres yellowish.

Abdomen blackish, grey dusted. Female segments 7-8 shining. Male sternite 4 approximatelly as wide as long. Male sternite 5 with narrow diverging lateral lobes each broaded inside basally (Fig. 12). Epandrium, cercal plate and surstyli as in Figs 31-33.

DISTRIBUTION. Russia: Dagestan, KarachayCherkessia, North Ossetia - Alania, Krasnodar Krai). - Abkhazia (first record), Georgia.

\section{Norellisoma lituratum (Meigen, 1826)} Figs 13, 34-36.

liturata Meigen, 1826: 382 (238) (Cordylura). Type-locality: not given.

flavicornis Meigen, 1826: 239 (Cordylura). Type-locality: not given ("auch einmal hier gefangen").

spinigera Zetterstedt, 1838: 733 (Cordylura). Type-locality: "Nordlandiae inferalpinus ... insula Hacksten prope Schiervoe... (Lappon. Norveg.)" (Norway).

altaica Ozerov, 2008: 2 (Norellia). Type-locality: $45 \mathrm{~km} \mathrm{E}$ of Ust'-Ulagan, the upper reaches of the Yoldu River $\left(50.5^{\circ} \mathrm{N} 88.57^{\circ} \mathrm{E}\right)$, 2020 m (Altai, Russia) — syn.n.

NOTE. Recorded as widespread species in Russia [Gorodkov, 1970, 1986].

MATERIAL. Altai: Aktash env. $\left(50.32^{\circ} \mathrm{N} 87.73^{\circ} \mathrm{E}\right), 2600 \mathrm{~m}$, 6.VII.2016, N. Vikhrev (6 $\sigma^{7} \sigma^{7}, 5$ 우, ZMUM); Seminsky pass, $\left(51.0^{\circ} \mathrm{N} 85.5^{\circ} \mathrm{E}\right), 1650 \mathrm{~m}, 27-30 . \mathrm{VI} .2016, \mathrm{~N}$. Vikhrev $\left(1 \sigma^{7}, 4\right.$ 9 , ZMUM); Seminsky pass, Sarlyk River $\left(51.1^{\circ} \mathrm{N} 85.6^{\circ} \mathrm{E}\right), 1200 \mathrm{~m}$, 28-30.VI.2016, N. Vikhrev (1 ㅇ, ZMUM); Kosh-Agach, Kurayskiy ridge, $2500-2700 \mathrm{~m},\left(50.3^{\circ} \mathrm{N} 87.7^{\circ} \mathrm{E}\right)$, 3.VII.2008, A. Barkalov (1 $\sigma^{7}$, ISEA); Arkhangelsk Oblast: Kil'din I., $\left(64.747^{\circ} \mathrm{N}\right.$ 40.314 ${ }^{\circ}$ E), 13.VII.1908, Bianki (1 $\sigma^{7}$, ZISP); Bashkiria: Makhmutovo env., Belaya River $\left(54.330^{\circ} \mathrm{N} 58.807^{\circ} \mathrm{E}\right), 550 \mathrm{~m}, 15 . \mathrm{VII} .2015$, D. Gavryushin (1 $\sigma^{7}$, ZMUM); Buryatia: Barguzinskiy reserve, Sosnovka env. (ca. $54.182^{\circ} \mathrm{N} 109.539^{\circ} \mathrm{E}$ ), 4.VIII.1962, Gorodkov 


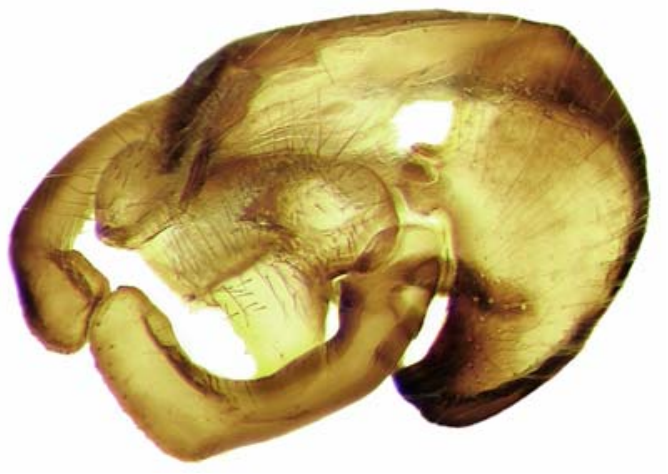

20
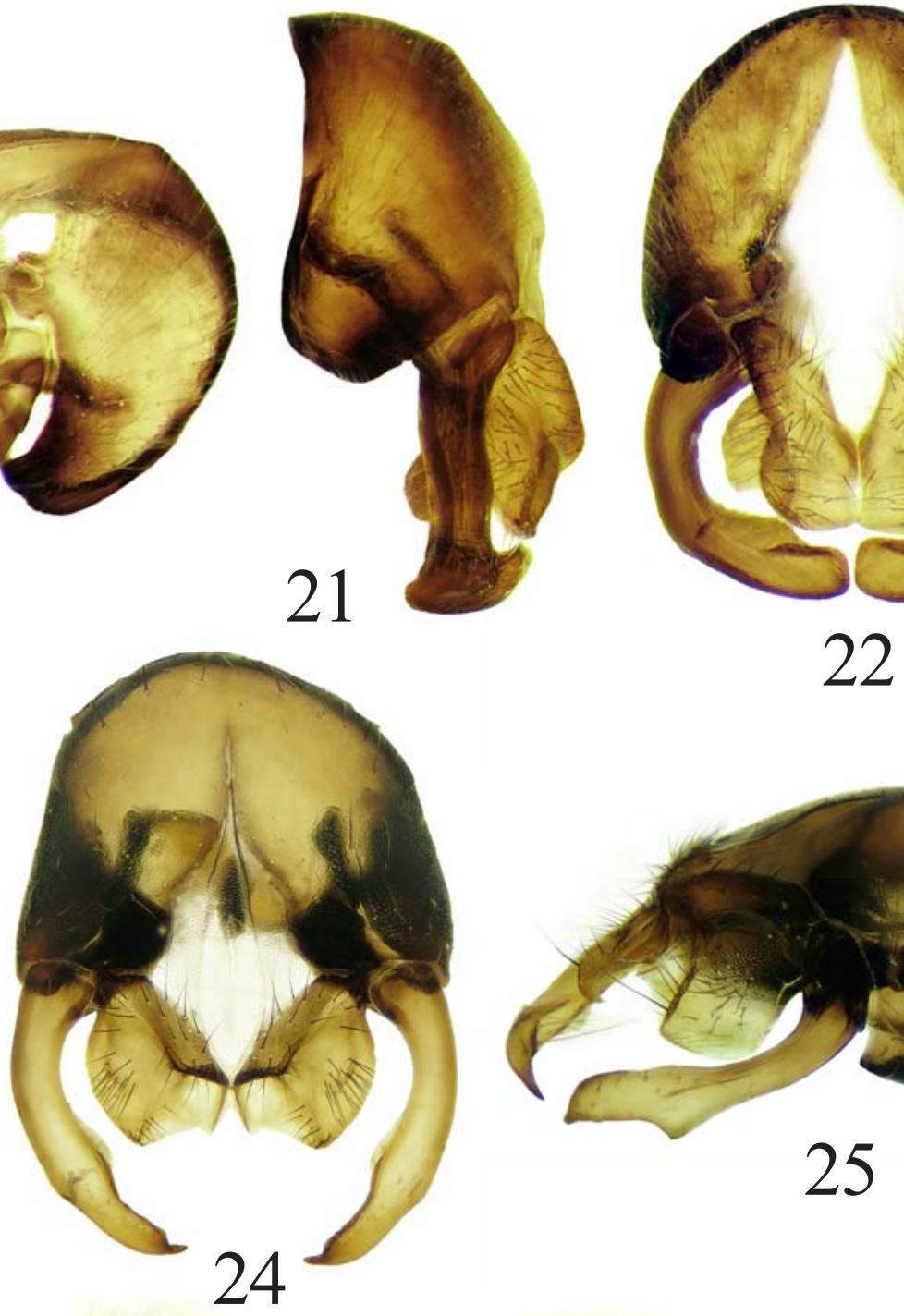

22

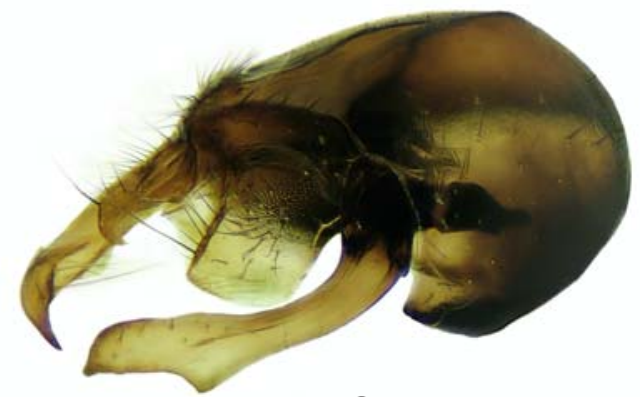

25
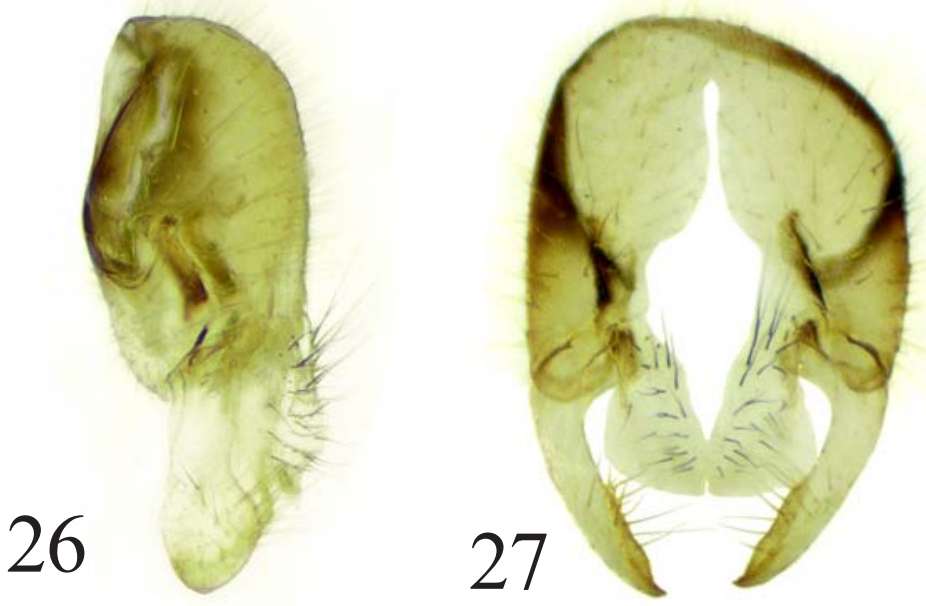

Figs 20-27. Epandrium, cercal plate and surstyli of Norellisoma spp.: 20-22 - N. armipes (Meigen); 23-25 - N. caucasicum (Ozerov); 26, 27 - N. flavostriatum Ozerov; 20, 25- dorsolateral view; 21, 23, 26 - lateral view; 22, 24, 27 - dorsal view. 20-22 after Ozerov, 2010a, figs 7-9; 25 - after Ozerov, 2010b, fig. 16; 26, 27 - after Ozerov, 2009, figs 16, 17.

Рис. 20-27. Эпандрий, церкальная пластинка и сурстили Norellisoma spp.: 20-22 — N. armipes (Meigen); 23-25 — N. caucasicum (Ozerov); 26, 27 - N. flavostriatum Ozerov; 20, 25 - дорсолатерально; 21, 23, 26 - сбоку; 22, 24, 27 - сверху. 20-22 — по Ozerov, 2010a, figs 7-9; 25 — по Ozerov, 2010b, fig. 16; 26, 27 — по Ozerov, 2009, figs 16, 17. 

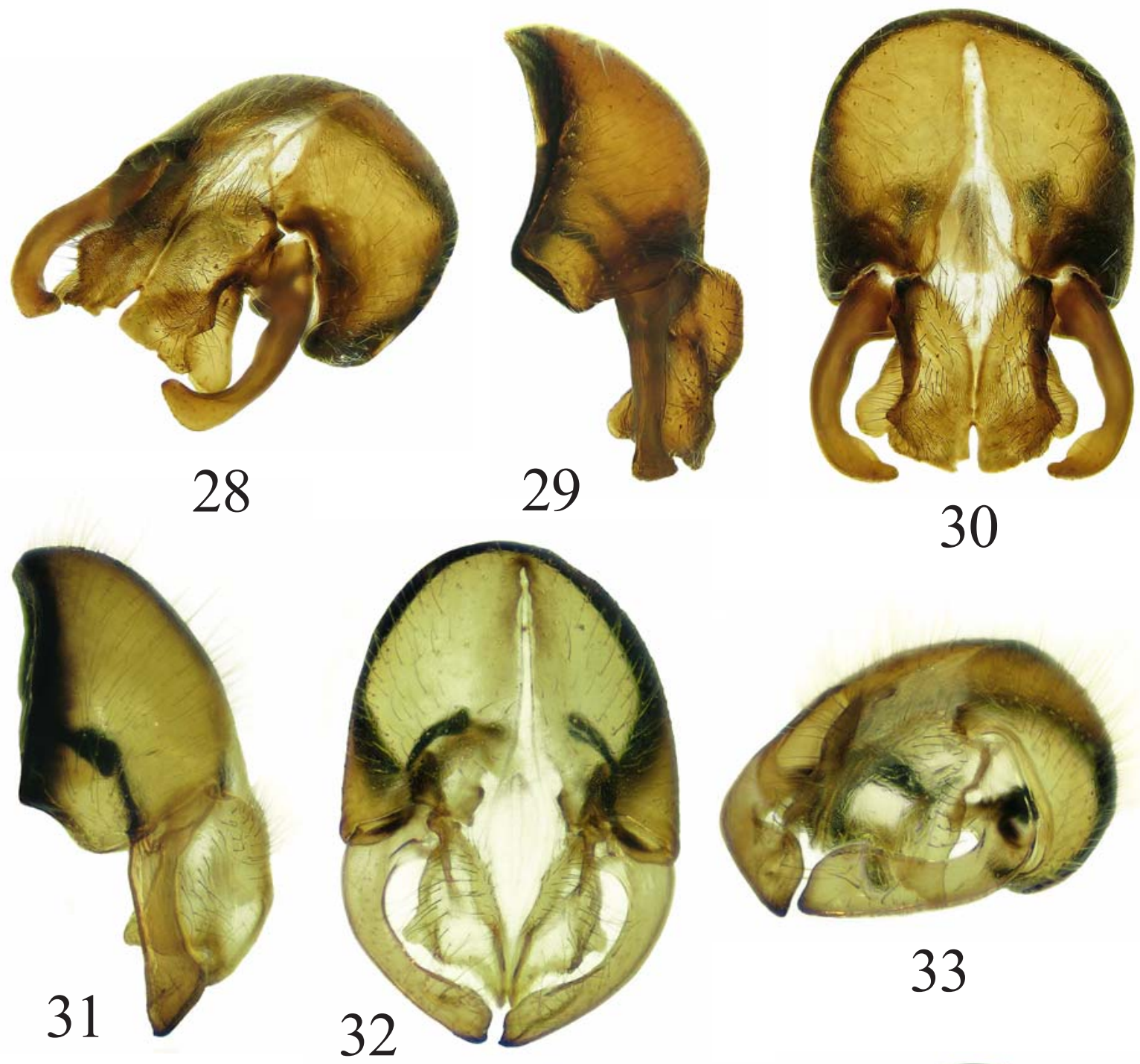

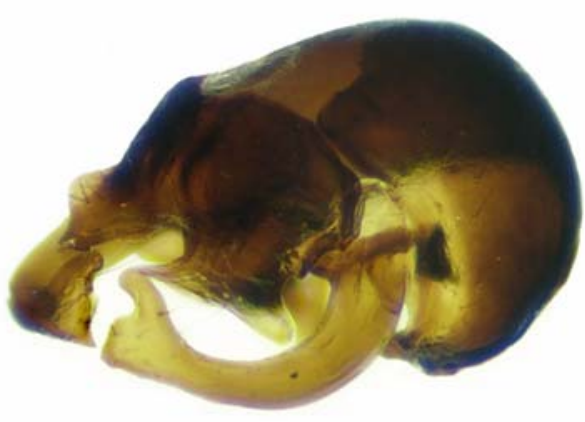

34
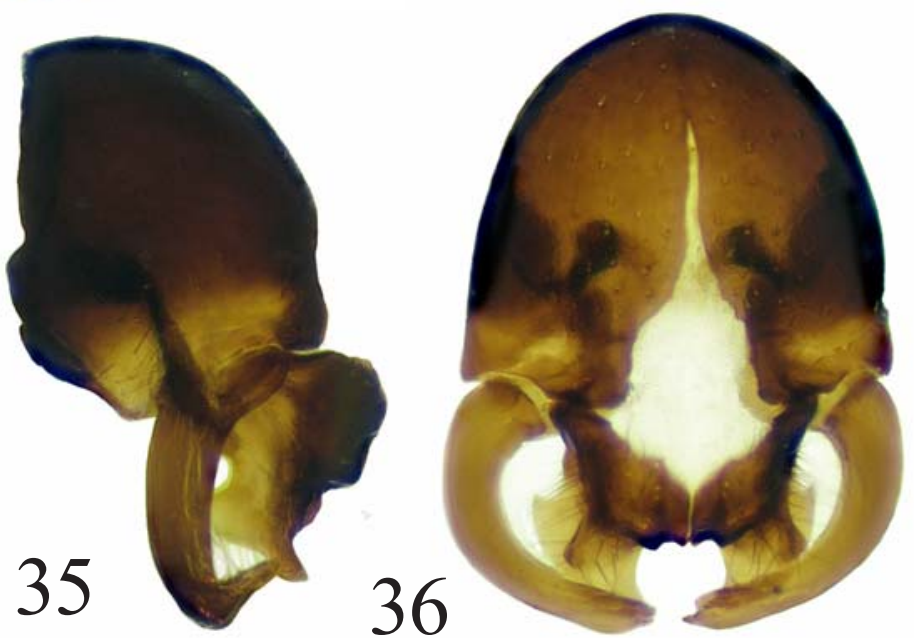

Figs 28-36. Epandrium, cercal plate and surstyli of Norellisoma spp.: 28-30-N. insulare (Ozerov); 31-33 - N. lesgiae (Becker); 34-36 - N. lituratum (Meigen); 28, 33, 34 - dorsolateral view; 29, 31, 35 - lateral view; 30, 32, 36 - dorsal view. 33, 34 - after Ozerov, 2010a, figs 25, 28.

Рис. 28-36. Эпандрий, церкальная пластинка и сурстили Norellisoma spp.: 28-30-N. insulare (Ozerov); 31-33 - N. lesgiae (Becker); 34-36 - N. lituratum (Meigen); 28, 33, 34 - дорсолатерально; 29, 31, 35 - сбоку; 30, 32, 36 - сверху. 33, 34 - по Ozerov, 2010a, figs 25, 28. 
(1 ㅇ, ZISP); Karachay-Cherkessia: Klukhor River (ca. $43.250^{\circ} \mathrm{N}$ 41.834 ${ }^{\circ} \mathrm{E}$ ), 2200 m, 25.VII.1905, Kalishevs[?kiy], (1 o', ZISP); Komi: Ust-Tsilma $\left(65.440^{\circ} \mathrm{N} 52.153^{\circ} \mathrm{E}\right), 4$.VI.1908, Zhuravskiy (1 $\sigma^{7}$ ZISP); Krasnodar Krai: Kryuchkovo station $\left(56.09^{\circ} \mathrm{N} 92.10^{\circ} \mathrm{E}\right), 14$ 24.VII.2009, K. Tomkovich (3 $\sigma^{7} \sigma^{7}, 5$ Oᄋ , ZMUM); Lagonaki $\left(44.0^{\circ} \mathrm{N}\right.$ $\left.39.9^{\circ} \mathrm{E}\right), 1500-1900 \mathrm{~m}, 5-9 . V I .2015$, N.Vikhrev (2 $\sigma^{7} \sigma^{7}$, ZMUM); Lagonaki $\left(44.10^{\circ} \mathrm{N} 40.02^{\circ} \mathrm{E}\right), 1450 \mathrm{~m}, 15-17 . V I .2009$, K. Tomkovich

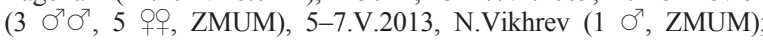
Estosadok, Psekhako Mt., (43.691 $\left.{ }^{\circ} \mathrm{N} 40.366^{\circ} \mathrm{E}\right),{ }_{14}-18 . V I .2008, \mathrm{~K}$ Tomkovich $\left(1 \mathrm{O}^{7}, \mathrm{ZMUM}\right)$; Teberdinskiy reserve $\left(43.447^{\circ} \mathrm{N} 41.741^{\circ} \mathrm{E}\right)$ 26.V.1968, Gorodkov (1 $\sigma^{7}$, ZISP); Krasnoyarsk Krai: Turuchansk $\left(65.797^{\circ} \mathrm{N} 87.958^{\circ} \mathrm{E}\right), 27 . V I .1967$, Gorodkov (1 ${ }^{7}, 1$ ㅇ, ZISP); Agapa (ca. $71.412^{\circ} \mathrm{N} 89.268^{\circ} \mathrm{E}$ ), Pyasina River, 19.VII.1967, Gorodkov (1 $q$, ZISP); Pyasina River (ca. $72.359^{\circ} \mathrm{N} 90.582^{\circ} \mathrm{E}$ ), $90 \mathrm{~km} \mathrm{~S}$ of Tareya, 23.VII.1967, Gorodkov (1 9 , ZISP); Leningrad Oblast: Otradnoe $\left(59.778^{\circ} \mathrm{N} 30.816^{\circ} \mathrm{E}\right), 6$. VI.1965, Gorodkov (1 9 , ZISP); Murmansk Oblast: Vudyavr Lake Basin (ca. $67.646^{\circ} \mathrm{N} 33.644^{\circ} \mathrm{E}$ ), 28.VII.1931, Fridolin ( $1 \sigma^{\prime}$, ZISP); Tuva: Mondy env. (51.675 $\left.\mathrm{N} 100.992^{\circ} \mathrm{E}\right), 19$ and 23.VII.1965, Gorodkov (3 $\sigma^{7} \sigma^{\top}, 1$, ZISP); Tyumen' Oblast: $\left(63.81^{\circ} \mathrm{N} 59.56^{\circ} \mathrm{E}\right), 1-8 . \mathrm{VII} .2010, \mathrm{~K}$. Tomkovich $\left(60^{7} \sigma^{7}, 3\right.$ 우,

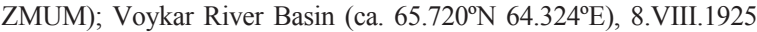
Fridolin (1 9 , ZISP); Sob' River Basin (ca. $66.937^{\circ} \mathrm{N} 65.670^{\circ} \mathrm{E}$ ), 18.VII.1925, Fridolin (1 $\sigma^{7}$, ZISP); Valley of the river Khadutte (ca. $\left.67.37^{\circ} \mathrm{N} 76.104^{\circ} \mathrm{E}\right), 83 \mathrm{~km} \mathrm{~W}$ of Tazovskoe, 30.VII.1977, Gorodkov (1 $\sigma^{7}$, ZISP); Labytnangi $\left(66.657^{\circ} \mathrm{N} 66.391^{\circ} \mathrm{E}\right), 23$. VII.1973, R. Kamenskaya ( $1 \sigma^{7}$, ZMUM)

ADDITIONAL MATERIAL. Mongolia: Kosogol Lake [=Khövsgöl] (ca. $\left.51.633^{\circ} \mathrm{N} 100.549^{\circ} \mathrm{E}\right), 19 . \mathrm{VI} .1880$, Potanin $\left(3 \sigma^{7} \sigma^{7}, 1\right.$, ZISP).

DESCRIPTION. Male, female. Medium-sized flies (5.0-7.8 $\mathrm{mm}$ long).

Head. Frontal vitta yellow; fronto-orbital plate yellow completely or blackish in upper half. Postcranium blackish in upper part and whitish in lower third. 2 frontal setae present. Antenna yellow. Postpedicel approximately 2 times as long as wide. Arista blackish, short haired: the length of hairs less (or equal) than the width of basal diameter of arista.

Thorax and scutellum black, grey dusted. Scutum with the following paired setae: (1-2)+2 dorsocentrals (anterior presutural seta small), $1+2$ supra-alars, 1 postpronotal, 1-2 notopleural (usually only anterior seta present, posterior seta always small), 2 postalars. Anepisternum with 1 seta near posterior margin.

Legs entirely yellow, only coxae of mid and hind legs partly darkened. Fore femur with 9-11 long posteroventral, 7-8 short anteroventral, 5-7 dorsal/posterodorsal setae. Fore tibia with 5 strong posteroventral and 5-7 strong anteroventral setae. Mid tibia with 1 anteroventral, 1 anterodorsal, 1-2 posterodorsal, 2-3 posteroventral and a ring of apical setae. Hind tibia with 1-3 anterodorsal, 1-2 posterodorsal, 1 preapical dorsal and 1 apical anteroventral setae; additionally with 1 anteroventral seta in apical quarter in female.

Wing tinged with brownish. Calypters, margins of calypters, and halteres yellowish.

Abdomen black, greyish dusted, often tergites shining laterally. Female segments $7-8$ shining. Male sternite 4 wider than long. Male sternite 5 with triangular lateral lobes usually strong divergent (Fig. 13). Epandrium, cercal plate and surstyli as in Figs 34-36. Surstylus with bifurcate apex (Fig. 34), the width of apical lobes varying in different specimens.

DISTRIBUTION. Russia: Altai, Arkhangelsk Oblast, Bashkiria, Buryatia, Karachay-Cherkessia, Kare- lia, Komi, Krasnodar Krai, Krasnoyarsk Krai, Leningrad Oblast, Murmansk Oblast, Tuva, Tyumen' Oblast. — Europe (widespread ) [Šifner, 2008], Mongolia [Gorodkov, 1986].

\section{Norellisoma montanopratense (Ozerov, 1993)} Figs 14, 37-39.

montanopratensis Ozerov, 1993: 67 (Norellia). Type-locality: $10 \mathrm{~km}$ SE of Alagir, Bakhty Lapparyrag ridge, $1600 \mathrm{~m}$ (North Ossetia - Alania, Russia)

MATERIAL. Krasnodar Krai: Kishi River $\left(44.057^{\circ} \mathrm{N}\right.$ 40.172 $\left.{ }^{\circ} \mathrm{E}\right)$, 18.VI.1911, Volnukhin (2 $\sigma^{7} \sigma^{7}$, ZISP); Estosadok, Psekhako Mt., (43.691 $\left.{ }^{\circ} \mathrm{N} 40.366^{\circ} \mathrm{E}\right), 14-18 . V I .2008$, K. Tomkovich $\left(1 \sigma^{7}, \mathrm{ZMUM}\right)$; Teberdinskiy reserve $\left(43.447^{\circ} \mathrm{N} 41.741^{\circ} \mathrm{E}\right), 25$ and 29.VI.1968, Gorodkov (2 O $\bigcirc^{7}, 2$ ㅇ, ZISP); North Ossetia Alania: Bakhty-Laparyrag ridge $\left(42.938^{\circ} \mathrm{N} 44.287^{\circ} \mathrm{E}\right), 1770 \mathrm{~m}$, 23.V.1989, A.L. Ozerov (1 $\sigma^{7}, 1$ ㅇ, ZMUM).

ADDITIONAL MATERIAL. Abkhazia: Klukhorskiy pass, valley of the river Klych (ca. $43.2441^{\circ} \mathrm{N} 41.8669^{\circ} \mathrm{E}$ ), 29.VI. and 2.VII.1968, Gorodkov (2 $0^{\top} \sigma^{7}$, ZISP);

DESCRIPTION. Male, female. Small or mediumsized flies (3.5-4.8 $\mathrm{mm}$ long).

Head. Frontal vitta yellow in lower half and blackish in upper half; fronto-orbital plate blackish. Postcranium black. 2-3 frontal setae present. Antenna blackish in ground color, pedicel and postpedicel partly reddish. Postpedicel approximately 2 times as long as wide. Arista blackish, short haired: the length of hairs less than the width of basal diameter of arista.

Thorax and scutellum black, grey dusted. Scutum with the following paired setae: $2+2$ dorsocentrals, $1+2$ supra-alars, 1 postpronotal, 1-2 notopleural (usually only anterior seta present, posterior seta always small), 2 postalars. Anepisternum with 1-3 setae near posterior margin.

Legs. Coxae and femora of all legs black; tibiae of all legs yellow or blackish; tarsi of all legs yellow. Fore femur with 8-9 long posteroventral, 6-7 short anteroventral, 5-7 dorsal/posterodorsal setae. Fore tibia with 4 strong posteroventral and 5-6 strong anteroventral setae. Mid tibia with 1 anterodorsal, 1-2 posterodorsal and a ring of apical setae in both sexes, additionally in female with $0-1$ anteroventral, 2-3 posteroventral setae. Hind tibia with 2 anterodorsal, 1-2 posterodorsal, 1 preapical dorsal and 1 apical anteroventral setae; additionally in female with 1 anteroventral seta in apical quarter.

Wing tinged with brownish. Calypters, margins of calypters, and halteres yellowish.

Abdomen black, grey dusted. Female segment 7 greyish dusted, segment 8 shining. Male sternite 4 wider than long. Male sternite 5 with narrow slightly divergent lateral lobes (Fig. 14). Epandrium, cercal plate and surstyli as in Figs 37-39.

DISTRIBUTION. Russia: Krasnodar Krai, North Ossetia - Alania. - Abkhazia (first record).

Norellisoma oreinum Ozerov, 2010 Figs 15, 40-42.

oreinum Ozerov, 2010a: 231 (Norellisoma). Type-locality: N Lagonaki Mt., Arish cave env. $\left(44.093^{\circ} \mathrm{N}, 40.019^{\circ} \mathrm{E}\right)$ (Adygea, Russia). 
MATERIAL. Adygea: Lagonaki $\left(44.09^{\circ} \mathrm{N} 40.01^{\circ} \mathrm{E}\right), 1725 \mathrm{~m}$ 26-28.VI.2009, K. Tomkovich (3 $\sigma^{7} \sigma^{7}, 1$ ㅇ, ZMUM); Lagonak $\left(44.0^{\circ} \mathrm{N} 39.9^{\circ} \mathrm{E}\right), 1500-1900 \mathrm{~m}, 5-9 . V I .2015$, N.Vikhrev $\left(2 \bigcirc^{7} \sigma^{7}\right.$, 4 우, ZMUM); Krasnodar Krai: Teberdinskiy reserve (ca. $\left.43.443^{\circ} \mathrm{N} 41.738^{\circ} \mathrm{E}\right), 13 . V .1964,21-26 . V I ., ~ 5 . V I I .1968$, Gorodkov

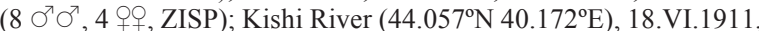
Volnukhin ( $1 \sigma^{\top}, 4$ 우, ZISP); Dzhuga Mt. $\left(43.888^{\circ} \mathrm{N} 40.410^{\circ} \mathrm{E}\right)$, 15-18.VI.1911, Volnukhin ( 7 O 9 , ZISP); Tsetse River (ca. $44.614^{\circ} \mathrm{N}$ $39.548^{\circ} \mathrm{E}$ ), 13 and 16.VI.1903, Filipchenko (2 $\sigma^{\top} \sigma^{\top}, 2$ 우, ZISP); Chekhashka Mt. env., 3.VI.1903, Filipchenko (1 $\sigma^{7}, 1$ \%, ZISP).

ADDITIONAL MATERIAL. Abkhazia: Bagri-Yashta ridge $\left(43.440^{\circ} \mathrm{N} 40.708^{\circ} \mathrm{E}\right), 2000 \mathrm{~m}, 2 . \mathrm{VII} .2009$, A. Gusakov $\left(3 \mathrm{O}^{\top} \mathrm{O}^{\top}, 2\right.$ o+ , ZMUM); Achibakh ridge $\left(43.4194^{\circ} \mathrm{N} 40.6263^{\circ} \mathrm{E}\right), 1830 \mathrm{~m}$, 24.VI.2009, A. Gusakov (2 $\sigma^{7} \sigma^{7}, 1$, ZMUM), same place $\left(43.4180^{\circ} \mathrm{N} 40.5860^{\circ} \mathrm{E}\right), 2240 \mathrm{~m}, 26 . V I .2009$, A. Gusakov (1 $\sigma^{7}$, ZMUM); ca. $2.3 \mathrm{~km} \mathrm{SE}$ of Chedym Mt. $\left(43.2938^{\circ} \mathrm{N} 41.0580^{\circ} \mathrm{E}\right)$, 1900 m, 1.VII.2012, A. Prosvirov (1 or, 1 , ZMUM).

DESCRIPTION. Male, female. Small or mediumsized flies (3.8-7.2 mm long).

Head. Frontal vitta yellow; fronto-orbital plate yellow in lower part and blackish in upper half or third. Postcranium black in upper part and yellow in lower 1/ 3 or 1/4. 2-3 frontal setae (usually 2 setae only) present. Antenna yellow, but postpedicel in female usually blackish on outside surface. Postpedicel approximately 2 times as long as wide. Arista black, short haired: the length of hairs about the width of basal diameter of arista.

Thorax and scutellum black, greyish dusted. Scutum with the following paired setae: $2+3$ dorsocentrals, $1+2$ supra-alars, 1 postpronotal, 2 notopleurals (posterior usually small), 2 postalars. Anepisternum with 1-2 setae near posterior margin.

Legs yellow in ground color, but often (usually in female) darkened dorsally. Male femora and tibiae with hairs longer than in female. Fore femur with 6-10 long posteroventral and 6-9 short anteroventral setae. Fore tibia with 4 strong posteroventral and 5-7 strong anteroventral setae. Mid tibia with 1 anteroventral, 1 anterodorsal, 1-2 posterodorsal and a ring of apical setae in both sexes, in male additionally with long pale hairs ventrally, in female with 2-3 posteroventral setae. Hind tibia with 2-3 anterodorsal, 2 posterodorsal, 1 preapical dorsal and 1 apical anteroventral setae.

Wing tinged with brownish. Calypters, margins of calypters, and halteres yellowish.

Abdomen blackish, greyish dusted. Female segments 7-8 subshining. Male sternite 4 wider than long. Male sternite 5 with narrow diverging lateral lobes (Fig. 15). Epandrium, cercal plate and surstyli as in Figs 40-42.

DISTRIBUTION. Russia: Adygea, Krasnodar Krai. - Abkhazia

\section{Norellisoma orientale (Ozerov, 1993)}

Figs 16, 43-45.

orientalis Ozerov, 1993: 70 (Norellia). Type-locality: town Zeya (Amur Oblast, Russia)

NOTE. Recorded by Ozerov \& Krivosheina [2014]

for Russia from Far East.

MATERIAL. Amur Oblast: Nora River basin, Sorokoverstnaya Channel $\left(52.528^{\circ} \mathrm{N} 129.965^{\circ} \mathrm{E}\right)$, 1.VIII.2006, E.M. Veselova, A.B. Ryvkin $\left(1\right.$ +, ZMUM); Selemzha River $\left(52.42119^{\circ} \mathrm{N} 129.93507^{\circ} \mathrm{E}\right)$ 8.VIII.2006, E.M. Veselova, A.B. Ryvkin (1 +, ZMUM); Zeyskiy re- serve, cordon "52 km" $\left(54.087^{\circ} \mathrm{N} 126.871^{\circ} \mathrm{E}\right)$, 10.VII.1981, A.L. Ozerov (1 , ZMUM); same place, 20.VII.1982, Zlobin (1 or, ZISP); Krasnoyarsk Krai: "Stolby" (55.96º 92.75E), 300 m, 28-31.VII.2009, K. Tomkovich (1 $\sigma^{\prime}$, ZMUM); Primorsky Krai: Bikin River, 22 km abobe the mouth of Svetlovodnaya River $\left(46.616^{\circ} \mathrm{N} 137.054^{\circ} \mathrm{E}\right)$, 14.VIII.1980, V. Zlobin, (1 $\sigma^{\top}$, ZISP); Kamenushka $\left(43.634^{\circ} \mathrm{N}\right.$ $\left.132.222^{\circ} \mathrm{E}\right), 16$ and 26.VII.1983, 15.VII.1984, A. Shatalkin (3 $\sigma^{7} \sigma^{7}, 1$ +, ZMUM); same place, 12.VII.1987, A.L. Ozerov (1 +, ZMUM); Lazovskiy reserve, cordon "Amerika" $\left(43.283^{\circ} \mathrm{N} 134.044^{\circ} \mathrm{E}\right), 13-$ 28.VII.1986, A.L. Ozerov (5 $\sigma^{7} \sigma^{7}$, ZMUM); riverhead of the Chapingou [= Krounovka] River, tributary of the Shufan [= Borisovka] River (ca. $\left.43.615^{\circ} \mathrm{N} 131.541^{\circ} \mathrm{E}\right), 1$. VII.1962, Nartshuk (1 $\sigma^{7}$, ZISP)

DESCRIPTION. Male, female. Medium-sized flies (6.1-8.2 mm long).

Head. Frontal vitta and fronto-orbital plate yellow. Postcranium yellow. 3-4 frontal setae present. Antenna yellow. Postpedicel approximately 2.5 times as long as wide. Arista blackish, moderate haired: the length of hairs more than the width of basal diameter of arista.

Thorax yellow, delicately greyish dusted. Scutum with two blackish stripes along dorsocentral lines and with the following paired setae: $2+3$ dorsocentrals, a pair of scapular setae (usually small), $1+2$ supra-alars, 1 postpronotal (rarely 2 setae), 2 notopleural, 2 postalars. Anepisternum with 1-2 setae near posterior margin. Scutellum yellow or blackish, delicately greyish dusted.

Legs entirely yellow, only mid and hind femora blackish dorsally near apex. Male femora and tibiae with hairs longer than in female. Fore femur with 8-10 long posteroventral and 7-9 short anteroventral setae. Fore tibia with 4 strong posteroventral and 5-6 strong anteroventral setae. Mid tibia with 1-2 anterodorsal, 1-2 posterodorsal and a ring of apical setae in both sexes, in male additionally with long pale hairs ventrally, in female with 1 anteroventral and 2-3 posteroventral setae. Hind tibia with 2-3 anterodorsal, 3-4 posterodorsal, 1 anteroventral, 1 preapical dorsal and 1 apical anteroventral setae.

Wing tinged with brownish. Calypters, margins of calypters, and halteres yellow.

Abdomen yellowish or blackish, delicately greyish dusted. Female segments 7-8 shining. Male sternite 4 wider than long. Male sternite 5 with narrow stick-like lateral lobes and two short median proejctions; length of median projection more than the distance between median projection and lateral lobe (Fig. 16). Epandrium, cercal plate and surstyli as in Figs 43-45.

DISTRIBUTION. Russia: Amur Oblast, Krasnoyarsk Krai, Primorsky Krai.

Norellisoma spinimanum (Fallén, 1819)

Figs 1, 2, 3, 5-7, 17, 46-48.

suilla Fabricius, 1794: 343 (Musca). Nomen oblitum. Typelocality: "Germania".

spinimana Fallén, 1819: 7 (Cordylura). Type-locality: "Scania" (Skåne, Sweden).

septentrionale Hendel, 1930: 2 (Norelliosoma). Type-locality: Petropavlovsk (Kamchatka, Russia).

NOTE. This species is widespread in Russia [Hendel, 1930; Gorodkov, 1970, 1986; Ovchinnikov, 2013; Ozerov, Krivosheina, 2014; Bagachanova et al., 2016]. 

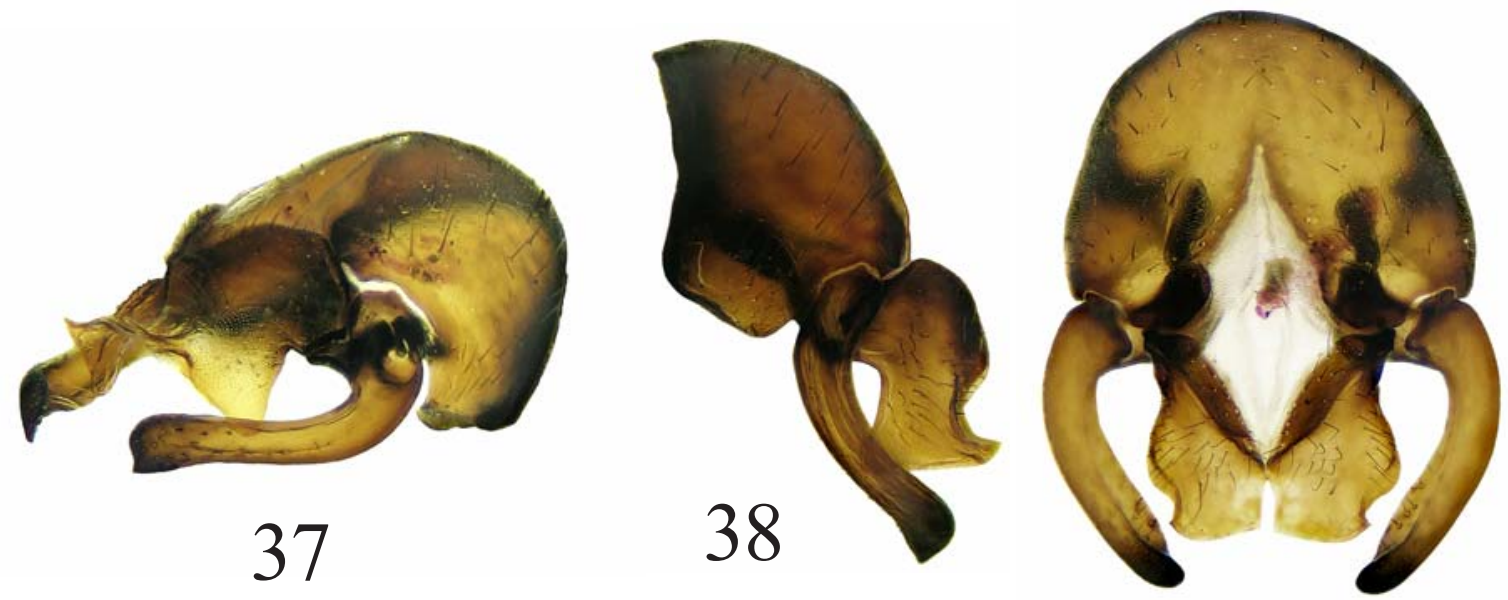

39
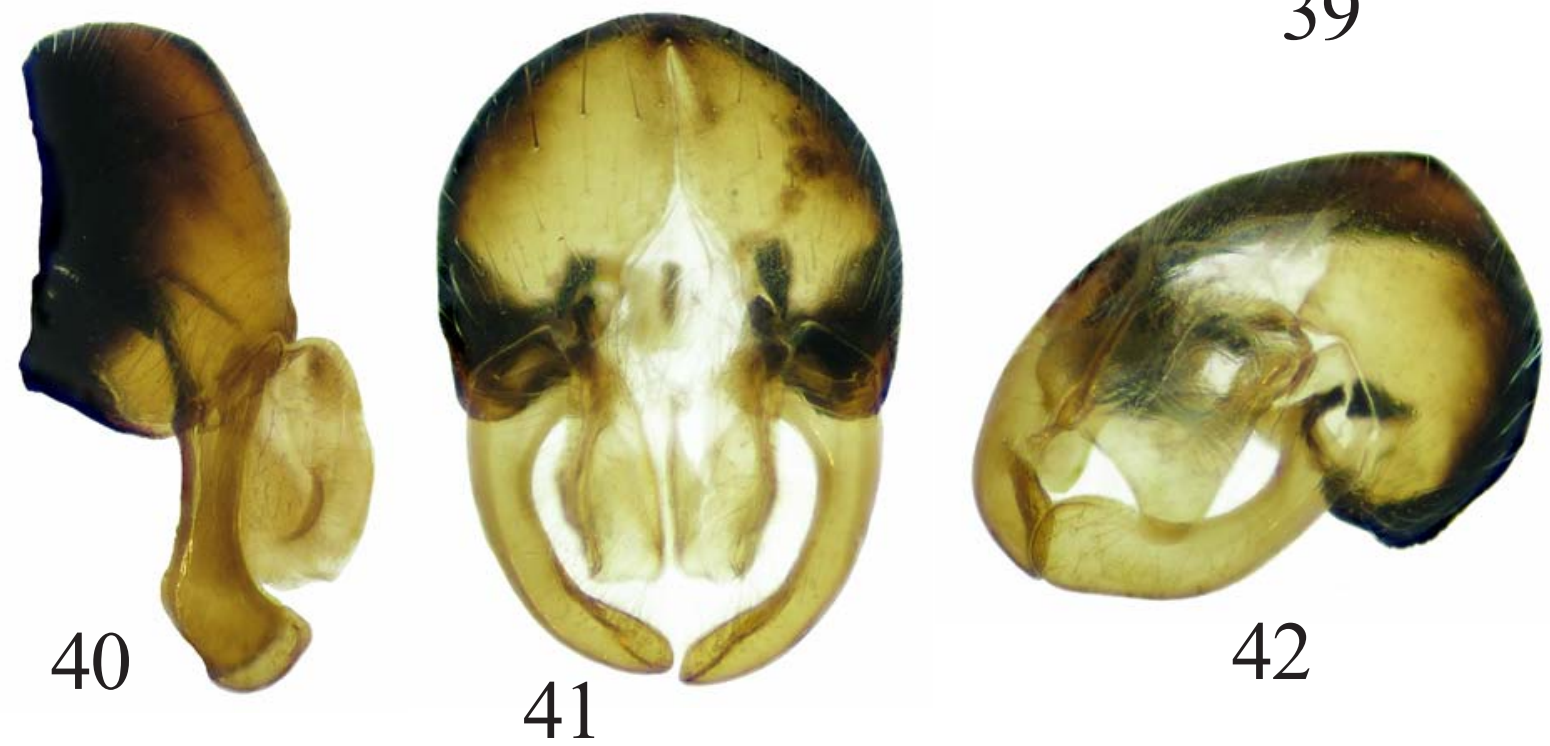

42
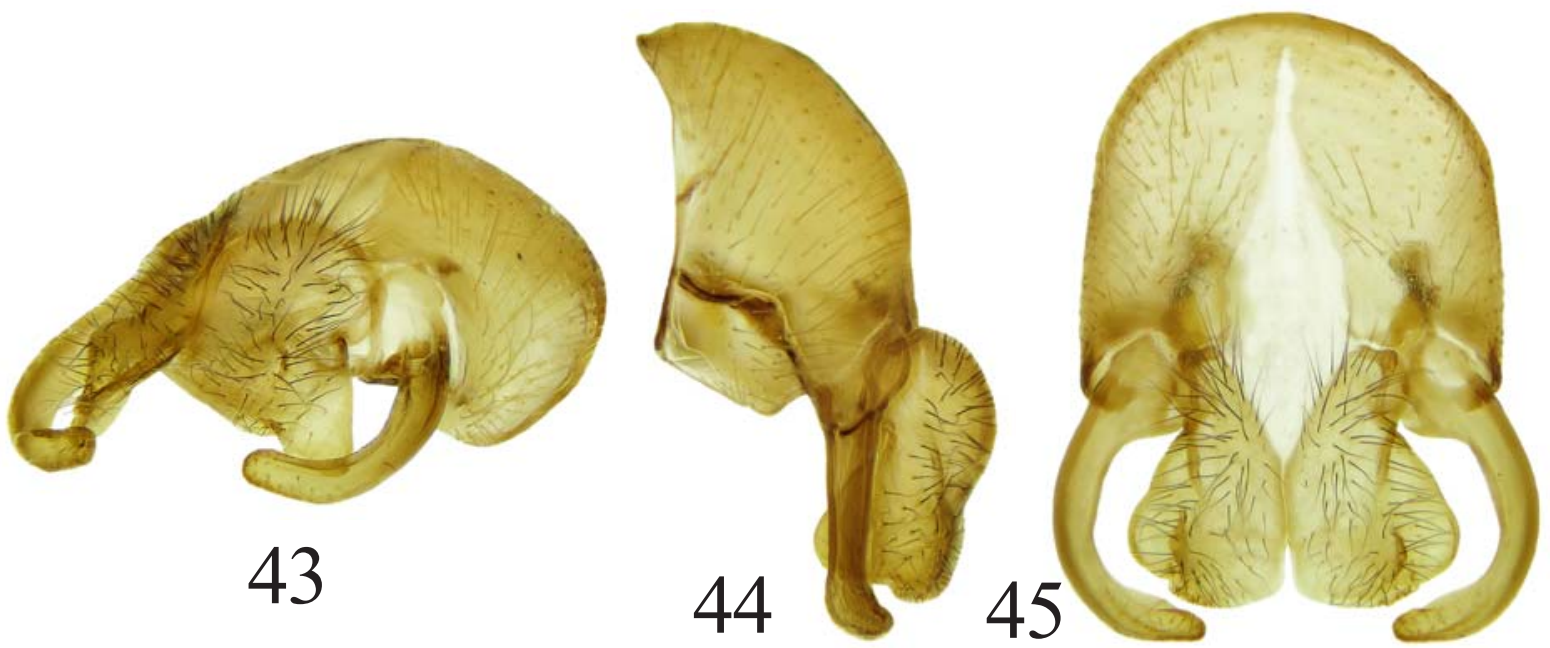

Figs 37-45. Epandrium, cercal plate and surstyli of Norellisoma spp.: 37-39-N. montanopratense (Ozerov); 40-42 - N. oreinum Ozerov; 43-45 - N. orientale (Ozerov); 37, 42, 43 - dorsolateral view; 38, 40, 44 - lateral view; $39,41,45-$ dorsal view. 40-42after Ozerov, 2010a, figs 19-21.

Рис. 37-45. Эпандрий, церкальная пластинка и сурстили Norellisoma spp.: 37-39-N. montanopratense (Ozerov); 40-42 - N. oreinum Ozerov; 43-45 - N. orientale (Ozerov); 37, 42, 43 - дорсолатерально; 38, 40, 44 — сбоку; 39, 41, 45 — сверху. 40-42 — по Ozerov, 2010a, figs 19-21. 

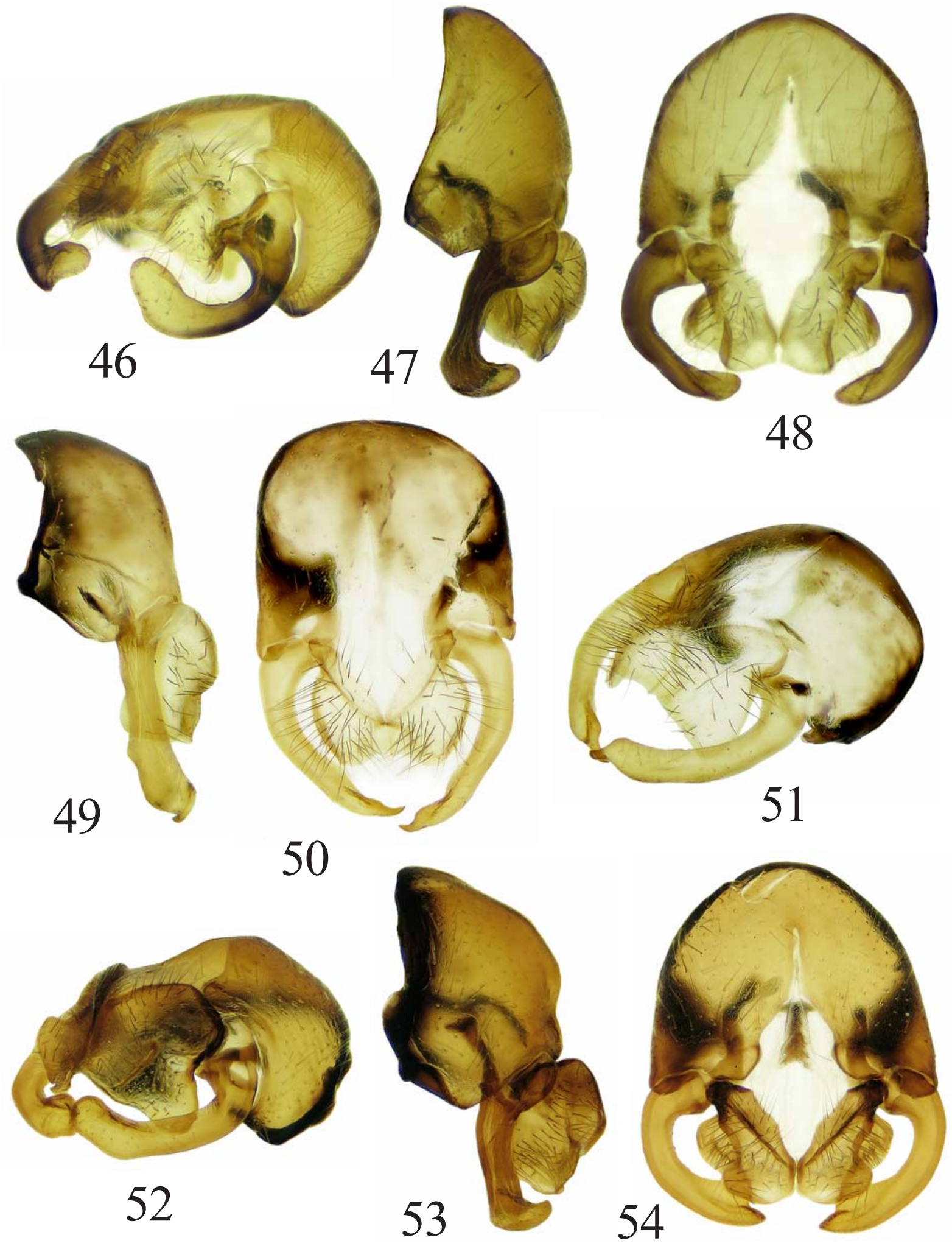

Figs 46-54. Epandrium, cercal plate and surstyli of Norellisoma spp.: 46-48 - N. spinimanum (Fallén); 49-51 - N. tomkovichi Ozerov; 52-54 - N. yolduense (Ozerov); 46, 51, 52 - dorsolateral view; 47, 49, 53 - lateral view; 48, 50, 54 - dorsal view. 46-48 after Ozerov, 2010a, figs 2-4; 49-51 — after Ozerov, 2010b, figs 10-12.

Рис. 46-54. Эпандрий, церкальная пластинка и сурстили Norellisoma spp.: 46-48 - N. spinimanum (Fallén); 49-51 - N. tomkovichi Ozerov; 52-54 - N. yolduense (Ozerov); 46, 51, 52 - дорсолатерально; 47, 49, 53 - сбоку; 48, 50, 54 - сверху. 46-48 — по Ozerov, 2010a, figs 2-4; 49-51 — по Ozerov, 2010b, figs 10-12. 
MATERIAL. Adygea: Guzeripl' $\left(43.998^{\circ} \mathrm{N} 40.1343^{\circ} \mathrm{E}\right)$ 23.VII.1935, Deev (1 $0^{7}$, ZISP); Lagonaki $\left(44.0^{\circ} \mathrm{N} 39.9^{\circ} \mathrm{E}\right), 1500-$ 1900 m, 5-9.VI.2015, N. Vikhrev (1 O7, 1 \%, ZMUM); Altai: Seminsky pass, $\left(51.0^{\circ} \mathrm{N} 85.5^{\circ} \mathrm{E}\right), 1629 \mathrm{~m}, 30 . \mathrm{VI} .2009$, A. Barkalov (1 $\mathrm{O}^{7}$, ISEA); same place, $1650 \mathrm{~m}, 27-30 . V I .2016$, N. Vikhrev (3 $\mathrm{O}^{7} \mathrm{O}^{7}$, ZMUM); Ust-Sema env., (51.6 $\left.6^{\circ} \mathrm{N} 85.8^{\circ} \mathrm{E}\right), 21-26 . V I .2016, \mathrm{~N}$. Vikhrev (1 ○', ZMUM); Artybash, $555 \mathrm{~m}\left(51.87^{\circ} \mathrm{N} 87.2^{\circ} \mathrm{E}\right), 21 . \mathrm{VI} .2009$, A Pont ( $1 \mathrm{O}^{7}$, UMO); Amur Oblast: Zeyskiy reserve, cordon " $52 \mathrm{~km}$ " $\left(54.087^{\circ} \mathrm{N} 126.871^{\circ} \mathrm{E}\right), 27 . V I .1982$, A.L. Ozerov (1 9 , ZMUM); Arkhangelsk Oblast: Karpogory (ca. $64^{\circ} \mathrm{N} 44.4^{\circ} \mathrm{E}$ ), River Pinega, 10.VII.1996, Gorodkov (1 9 , ZISP); the lower reaches of the Pechora River $\left(68.334^{\circ} \mathrm{N} 53.304^{\circ} \mathrm{E}\right), 11$. VII.2008, A.L. Ozerov (3 $\mathrm{O}^{7} \mathrm{O}^{7}, 1$ 웅, ZMUM); Bashkiria: Bashkirskiy reserve, Sargaya $\left(53.344^{\circ} \mathrm{N}\right.$ $\left.57.783^{\circ} \mathrm{E}\right)$, 7.VIII.1975, Gorodkov (1 , Z, ZISP); Buryatia: Khamney $\left(50.40^{\circ} \mathrm{N} 103.868^{\circ} \mathrm{E}\right), 29 . \mathrm{VI} .1971$, V. Richter (1 $0^{7}$, ZISP); Chelyabinsk Oblast: $63 \mathrm{~km}$ NNW of Chelyabinsk $\left(55.800^{\circ} \mathrm{N} 61.033^{\circ} \mathrm{E}\right), 17$ 19.V.1992, M. Krivosheina (1 $0^{7}$, ZMUM); Chuvashia: Yadrin $\left(55.8^{\circ} \mathrm{N}\right.$

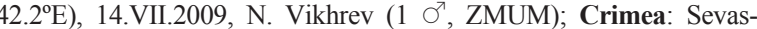
topol' $\left(44.567^{\circ} \mathrm{N} 33.540^{\circ} \mathrm{E}\right)$, without data, Pliginski (1 $\sigma^{\prime}$, ZISP); Simferopol' env. $\left(44.951^{\circ} \mathrm{N} 34.115^{\circ} \mathrm{E}\right)$, 29. IV.1899, Bazhenov (2 $\sigma^{7} \sigma^{\top}$, ZISP); Dagestan: $15 \mathrm{~km}$ SW of Sergokala, 15.VII.1983, Narchuk ( 1 ㅇ, ZISP); Irkutsk Oblast: Listvyanka $\left(51.863^{\circ} \mathrm{N} 104.866^{\circ} \mathrm{E}\right)$, 26.VI.1965, Negrobov (1 $\bigcirc^{7}$, ZISP); Nikola $\left(51.895^{\circ} \mathrm{N} 104.819^{\circ} \mathrm{E}\right)$, 24.VI.1965, Negrobov (1 $\sigma^{\prime}$, ZISP); Kaliningrad Oblast: Kurshskaya Kosa $\left(55.154^{\circ} \mathrm{N} 20.857^{\circ} \mathrm{E}\right)$, without data, V. Kolyda $\left(1 \mathrm{O}^{7}, 1\right.$ 웅 ZMUM); Kamchatka: Elizovo (53.187 $\left.\mathrm{N} 158.381^{\circ} \mathrm{E}\right), 11 . \mathrm{VIII} .1970$ Negrobov (1 $\sigma^{7}$, ZISP); Karelia: Poyakonda $\left(66.589^{\circ} \mathrm{N} 32.821^{\circ} \mathrm{E}\right)$, 8.VII.2010, A.L. Ozerov (1 $\sigma^{7}$, ZMUM); Primorskiy $\left(66.552^{\circ} \mathrm{N}\right.$ $33.100^{\circ}$ E), 7.VII.2010, A.L. Ozerov (1 +, ZMUM); Kemerovo Oblast: Tisul' $\left(55.759^{\circ} \mathrm{N} 88.310^{\circ} \mathrm{E}\right), 16$. VI.1911, Gorchakovskiy $(1 \%$ ZISP); Khabarovsk Krai: Khicha River $\left(49.0^{\circ} \mathrm{N} 139.4^{\circ} \mathrm{E}\right), 690 \mathrm{~m}$, 10.VI.2014, N. Vikhrev (1 + , ZMUM); Vanino $\left(49.1^{\circ} \mathrm{N} 140.3^{\circ} \mathrm{E}\right)$, 9.VI.2014, N. Vikhrev (1 $\mathrm{O}^{\top}, 2$ 우, ZMUM); Malyy Khekhtsir ridge $\left(48.257^{\circ} \mathrm{N} 135.191^{\circ} \mathrm{E}\right)$, 26.VIII.1979, Narchuk (1 $\circ$, ZISP); Komi Vorkuta $\left(67.492^{\circ} \mathrm{N} 64.072^{\circ} \mathrm{E}\right), 19-25$. VII.2010, N. Vikhrev (1 O7, ZMUM); Krasnodar Krai: Dakhovskaya env. $\left(44.19^{\circ} \mathrm{N} 40.17^{\circ} \mathrm{E}\right)$, 465 m, 18-31.VIII.2009, K. Tomkovich (1 ○7, ZMUM); Estosadok, Psekhako Mt., (43.691 $\left.{ }^{\circ} \mathrm{N} 40.366^{\circ} \mathrm{E}\right), 14-18 . V I .2008, \mathrm{~K}$. Tom-kovich (2 $ᄋ \circ$, ZMUM); Teberdinskiy reserve, valley of the Dzhamagat River $\left(43.470^{\circ} \mathrm{N} 41.745^{\circ} \mathrm{E}\right), 7 . V I I .1984$, Ovchinni-kova (1 $0^{7}$, ZISP); Teberdinskiy reserve, valley of the Teberda River $\left(43.405^{\circ} \mathrm{N} 41.721^{\circ} \mathrm{E}\right)$, 17.VII.1982, Narchuk (1 $0^{7}$, ZISP); Teberdinskiy reserve $\left(43.443^{\circ} \mathrm{N}\right.$ $\left.41.738^{\circ} \mathrm{E}\right), 10$ and 20.V.1964, 20.VI.-9.VII.1968, Gorodkov (2 $0^{7} \mathrm{O}^{7}$, 1 ․ ZISP); Krasnoyarsk Krai: "Stolby" $\left(55.96^{\circ} \mathrm{N} 92.73^{\circ} \mathrm{E}\right), 30$ 31.VII.2009, K. Tomkovich (1 9 , ZMUM); Turuchansk $\left(65.797^{\circ} \mathrm{N}\right.$ $\left.87.958^{\circ} \mathrm{E}\right), 29$. VI.1967, Gorodkov (2 $0^{7} \mathrm{O}^{7}$, ZISP); Kryuchkovo station $\left(56.09^{\circ} \mathrm{N} 92.10^{\circ} \mathrm{E}\right), 14-23 . \mathrm{VII} .2009,13-15 . \mathrm{VI} .2011, \mathrm{~K}$. Tomkovich ( 7 O $^{7} \mathrm{O}^{7}, 1$ \% , ZMUM); Kursk Oblast: Oboyan' $\left(51.191^{\circ} \mathrm{N} 36.312^{\circ} \mathrm{E}\right)$ 26.V. 2007, N. Vikhrev (1 $\sigma^{7}$, ZMUM), same place, 20-21.VII.2007, A.L. Ozerov $\left(1 \odot^{7}, 1\right.$ \&, ZMUM); Leningrad Oblast: Luga (ca. $\left.58.7^{\circ} \mathrm{N} 29.8^{\circ} \mathrm{E}\right), 1$ 2.VIII.1952, 13.VIII.1953, 15.VI.1954, 16.VII.1954, A. Stackelberg $\left(20^{7} \sigma^{7}, 2\right.$ 우, ZISP); Bol'shoy Berezovyy I. $\left(60 .^{\circ} \mathrm{N}\right.$ $\left.28.6^{\circ} \mathrm{E}\right)$, 24.VIII.1979, Kandybina (1 $\mathrm{O}^{\mathrm{T}}$, ZISP); Volkovitsy $\left(59.64^{\circ} \mathrm{N}\right.$ $\left.29.825^{\circ} \mathrm{E}\right)$, 11.VI.1946, Rubtsov (1 + , ZISP); Ligovo $\left(60.223^{\circ} \mathrm{N}\right.$ $31.797^{\circ}$ E), 3.VIII.1903, G. Yakobson (1 ․, ZISP); Luga (ca. 58.73N 29.84E), 16.VII.1954, 13.VI.1955, À. Stackelberg (2 $\sigma^{7} \sigma^{7}$, ZISP); Petergof $\left(59.889^{\circ} \mathrm{N} 29.897^{\circ} \mathrm{E}\right), 12$.VII.1895, A. Chekini (1 $\mathrm{O}^{\prime}$, ZISP); Rakovichi $\left(58.650^{\circ} \mathrm{N} 29.836^{\circ} \mathrm{E}\right), 10 . V .1897$, G. Pleske (1 $\sigma^{\top}$, ZISP); Terioki [ $=$ Zelenogorsk] $\left(60.195^{\circ} \mathrm{N} 29.699^{\circ} \mathrm{E}\right)$ [without data], Yu Vagner (1 $\mathrm{O}^{7}$, ZISP); Trubnikov Bor $\left(59.26^{\circ} \mathrm{N} 31.391^{\circ} \mathrm{E}\right), 21 . V .1967$ Gorodkov (1 $0^{7}$, ZISP); Yashchera $\left(58.894^{\circ} \mathrm{N} 29.820^{\circ} \mathrm{E}\right)$, 21.VIII.1962, 18.VIII.1965, 3.VII.1967, A. Stackelberg (1 O', 2 우, ZISP); Lipetsk Oblast: Ryazanka $\left(53.500^{\circ} \mathrm{N} 39.575^{\circ} \mathrm{E}\right), 1881$, A.I. Yakovlev (2 우, ZISP); Magadan Oblast: $\left(55.568^{\circ} \mathrm{N} 150.809^{\circ} \mathrm{E}\right), 18 . \mathrm{VI} .1963$, Zhelokhovtsev (1 $\mathrm{O}^{\mathrm{T}}$, ZMUM); Sokol env. (59.9 $\left.9^{\circ} \mathrm{N} 150.7^{\circ} \mathrm{E}\right), 11-$ 19.VII.2014, N. Vikhrev (1 9 , ZMUM); Mari EI Republic: YoshkarOla $\left(56.633^{\circ} \mathrm{N} 47.887^{\circ} \mathrm{E}\right), 14$. VI.1976, Karlova (1 $\mathrm{O}^{7}$, ZISP); Moscow and Moscow Oblast: Smolevo $\left(55.626^{\circ} \mathrm{N} 38.964^{\circ} \mathrm{E}\right), 10-20 . X .2009$, K. Tomkovich ( 1 ㅇ, ZMUM); Andreevskoe $\left(55.974^{\circ} \mathrm{N} 35.603^{\circ} \mathrm{E}\right)$, 4.VIII.2005, 16.VII.2006, 27.VI.2007, A.L. Ozerov ( $80^{7} \sigma^{7}$, ZMUM); Bittsa $\left(55.641^{\circ} \mathrm{N} 37.570^{\circ} \mathrm{E}\right), 24$. VI.1936, B. Rodendorf (1 $\mathrm{O}^{\prime}$, ZMUM) Bol'shevo $\left(55.925^{\circ} \mathrm{N} 37.868^{\circ} \mathrm{E}\right), 10 . \mathrm{V} .1930, \mathrm{~N}$. Violovich $\left(2 \mathrm{O}^{7} \mathrm{O}^{7}, 2\right.$ 우, ZMUM); Burtsevo $\left(55.981^{\circ} \mathrm{N} 35.598^{\circ} \mathrm{E}\right), 20$ and 23.V.2006,
10.VII.2007, 29.V.2010, A.L. Ozerov (4 $\sigma^{7} \sigma^{7}, 1$ ㅇ, ZMUM); Vel'yaminovo $\left(55.190^{\circ} \mathrm{N} 37.876^{\circ} \mathrm{E}\right), 8$.VI. and 14.VII.1987, A. Antropov $\left(2 \sigma^{7} \sigma^{7}, 2\right.$ 우, ZMUM); Govorovo $\left(55.651^{\circ} \mathrm{N} 37.429^{\circ} \mathrm{E}\right)$, 24.V.1980, G. Lyubarskiy (1 ㅇ, ZMUM); Golitsyno $\left(55.649^{\circ} \mathrm{N}\right.$ $\left.37.011^{\circ} \mathrm{E}\right), 26 . V I .1976,14 . V .1977,17$ and 23.V.1979, 24.V.14.VI.1981, 30.VII.1988, A. Shatalkin (1 $\sigma^{7}$, ZMUM); Egor'evsk $\left(55.372^{\circ} \mathrm{N} 39.042^{\circ} \mathrm{E}\right), 21 . \mathrm{VII} .1972$, A. Shatalkin ( $\left.1 \mathrm{O}^{7}, \mathrm{ZMUM}\right)$; Zele$\operatorname{nograd}\left(55.986^{\circ} \mathrm{N} 37.202^{\circ} \mathrm{E}\right), 29$.VIII. 1994, A. Gusakov (1 $\left.\mathrm{O}^{7}, \mathrm{ZMUM}\right)$; Izmaylovo $\left(55.800^{\circ} \mathrm{N} 37.832^{\circ} \mathrm{E} ; 55.794^{\circ} \mathrm{N} 37.832^{\circ} \mathrm{E} ; 55.786^{\circ} \mathrm{N}\right.$ $37.835^{\circ} \mathrm{E}$ ), 17-30.V.1986, 3.VI.1990, 31.V.1992, 3.VIII.1993, 17.VII.1996, 16-20.VI.2007, 12.VI.2009, A.L. Ozerov (14 $0^{7} \sigma^{7}, 6$ Oᄋ, ZMUM); Kuntsevo $\left(55.720^{\circ} \mathrm{N} 37.473^{\circ} \mathrm{E}\right)$, 5.VI.1982, A.L. Ozerov

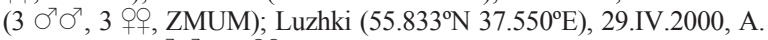

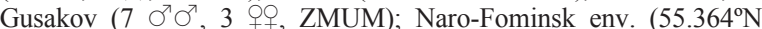
$\left.36.741^{\circ} \mathrm{E}\right), 11-13 . \mathrm{V} .2008,28 . \mathrm{V} .2009,2$ and 24.VIII.2009, 15.V.2010, 23.VI. and 2.VIII.2011, D. Gavryushin (5 $0^{7} \sigma^{7}, 5$ 우, ZMUM); NaroFominsk env. $\left(55.389^{\circ} \mathrm{N} 36.72^{\circ} \mathrm{E}\right), 29 . \mathrm{VII} .2014$, D. Gavryushin $(1$, ZMUM); Volokolamsk env. (55.983 $\left.{ }^{\circ} \mathrm{N} 35.616^{\circ} \mathrm{E}\right)$, 4.VIII.2005, A.L. Ozerov (1 $\bigcirc^{7}, 1$ \%, ZMUM); Zveni-gorod env. $\left(55.700^{\circ} \mathrm{N} 36.722^{\circ} \mathrm{E}\right)$, 21.VII.1981, G. Dlusskiy (1 $\sigma^{7}$, ZMUM); Ostankino $\left(55.828^{\circ} \mathrm{N}\right.$ $\left.37.612^{\circ} \mathrm{E}\right)$, 30.V.1904, F.K. (1 $\sigma^{\prime}$, ZMUM); Stepan'kovo $\left(55.996^{\circ} \mathrm{N}\right.$ $\left.35.615^{\circ} \mathrm{E}\right)$, 4.VII.2006, A.L. Ozerov (1 $\mathrm{O}^{7}$, ZMUM); Strogino $\left(51.786^{\circ} \mathrm{N}\right.$ $\left.37.414^{\circ} \mathrm{E}\right), 27$. VII.2009, N. Vikhrev (1 $0^{7}$, ZMUM); Skhodnya $\left(55.842^{\circ} \mathrm{N} 37.412^{\circ} \mathrm{E}\right)$, 4.VIII.1937, B. Rodendorf $\left(7 \mathrm{O}^{\top} \sigma^{7}, 4\right.$ q9, ZMUM); Tsaritsyno $\left(55.616^{\circ} \mathrm{N} 37.683^{\circ} \mathrm{E}\right), 6-10 . \mathrm{V} .2009, \mathrm{~K}$. Tomkovich (1 9 , ZMUM); Anikeevka $\left(55.827^{\circ} \mathrm{N} 37.214^{\circ} \mathrm{E}\right)$, 23.VII.1983, Gorodkov (2 $\bigcirc^{7} \sigma^{7}$, ZISP); Murmansk Oblast: Tyuva bay $\left(69.194^{\circ} \mathrm{N}\right.$ $\left.33.615^{\circ} \mathrm{E}\right), 20$ VI.1910, A. D'yakonov (1 $\sigma^{\top}, 1$ ㅇ, ZISP); Murmansk: $\left(68.9^{\circ} \mathrm{N} 33.0^{\circ} \mathrm{E}\right)$, 31.VIII.1981, Gorodkov (1 $\mathrm{O}^{\top}$, ZISP); Nizhegorod Oblast: Dzerzhinsk $\left(56.2^{\circ} \mathrm{N} 43.6^{\circ} \mathrm{E}\right), 16-18 . V I I I .2009$, N. Vikhrev (1 $\left.\mathrm{O}^{7}, \mathrm{ZMUM}\right)$; North Ossetia - Alania: Alagir env. $\left(43.015^{\circ} \mathrm{N} 44.224^{\circ} \mathrm{E}\right)$, 26.VII.1988, 16 and 24.VI.1990, A.L. Ozerov (2 $0^{7} \sigma^{7}, 1$, , ZMUM); Buron env. (43.793 $\left.{ }^{\circ} \mathrm{N} 43.922^{\circ} \mathrm{E}\right), 30 . \mathrm{VII} .1988,6$.VI.1989, 3.VII.1990, A.L. Ozerov (4 $0^{7} 0^{7}, 1$, ZMUM); same place, 16.IX.1988, A Shatalkin $\left(1 \mathrm{O}^{7}, 1\right.$ \%, ZMUM); Bakhty-Laparyrag ridge $\left(42.938^{\circ} \mathrm{N}\right.$ $\left.44.287^{\circ} \mathrm{E}\right), 1770 \mathrm{~m}, 31 . V .-1 . V I .1989$, A.L. Ozerov (4 $\mathrm{O}^{7} 0^{7}$, ZMUM); Novgorod Oblast: Tigoda $\left(59.358^{\circ} \mathrm{N} 31.809^{\circ} \mathrm{E}\right)$, 5.IX.1906, Semenov Tyan'-Shanskiy (1 $\mathrm{O}^{\prime}$, ZISP); Novosibirsk Oblast: $4 \mathrm{~km} \mathrm{~S}$ of Novososedovo $\left(54.616^{\circ} \mathrm{N} 83.985^{\circ} \mathrm{E}\right)$, 7.VII.2008, O. Koste-rin (1 ㅇ, ZMUM); Novosibirsk $\left(54.991^{\circ} \mathrm{N} 82.927^{\circ} \mathrm{E}\right), 2-8$.VIII.2008, O. Kosterin $\left(10^{7}\right.$, ZMUM); Omsk env. $\left(54.980^{\circ} \mathrm{N} 73.403^{\circ} \mathrm{E}\right)$, VII.1912, P. Ryabov (1 $\circ$, ZISP); Orenburg Oblast: Dneprovka $\left(51.424^{\circ} \mathrm{N}\right.$ $\left.56.345^{\circ} \mathrm{E}\right)$, 7.VIII.1935, L. Zimin (1 $\bigcirc^{\mathrm{T}}$, ZISP); Tsvillinga station

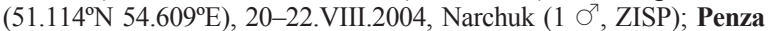
Oblast: Kuznetsk $\left(53.110^{\circ} \mathrm{N} 46.600^{\circ} \mathrm{E}\right), 5 . V I .1907, \mathrm{~N}$. Ikonnikov (1 + , ZMUM); Primorsky Krai: Kedrovaya Pad' reserve $\left(43.104^{\circ} \mathrm{N}\right.$ $\left.131.512^{\circ} \mathrm{E}\right)$, 6.VIII.1962, Nartshuk (1 9, ZISP); same place, 816.VI.2007, A. Ovchinnikov (3 ○ $^{7}$, 1 ㅇ, ZISP); Kamenushka $\left(43.618^{\circ} \mathrm{N} 132.229^{\circ} \mathrm{E}\right), 14 . V I I .1981$, Kasparyan (1 9 , ZISP); Pskov Oblast: Kharlamov Mt. $\left(58.713^{\circ} \mathrm{N} 28.713^{\circ} \mathrm{E}\right), 3 . V I .1891$, Pleske (1 $ᄋ$, ZISP); Ryazan' Oblast: Zalesnoe (54.96 $\left.\mathrm{N} 41.32^{\circ} \mathrm{E}\right), 21-26 . V I I .2013$, $\mathrm{N}$. Vikhrev $\left(1 \mathrm{O}^{7}, 1\right.$ \% , ZMUM); Gremyachka $\left(53.92^{\circ} \mathrm{N} 39.516^{\circ} \mathrm{E}\right)$, 30.V.1908, A. Semenov ( $1 \sigma^{7}$, ZISP); Smolensk Oblast: National Park "Smolenskoe poozer'e" (55.511 $\left.{ }^{\circ} \mathrm{N} 31.839^{\circ} \mathrm{E}\right)$, 8.VI.1992, Zlobin (1 9 , ZISP); Tatarstan: Volzhsko-Kamskiy reserve, Raifa Lake $\left(55.897^{\circ} \mathrm{N} 48.733^{\circ} \mathrm{E}\right), 8 . V I .1982,5 . V .1985$, Basov (3 $\sigma^{7} \sigma^{7}, 1$ q, ZMUM); Tula Oblast: Aleksin $\left(54.517^{\circ} \mathrm{N} 37.094^{\circ} \mathrm{E}\right), 16$.VII. 1899, V. Bezval' (1 $\sigma^{7}, 1$ O , ZISP); Fedyukino $\left(54.507^{\circ} \mathrm{N} 37.723^{\circ} \mathrm{E}\right)$, 22.VI.1903, Zhukov ( 1 \%, ZMUM); Tuva: Mondy env. $\left(51.675^{\circ} \mathrm{N}\right.$ $\left.100.992^{\circ} \mathrm{E}\right), 24$.VII.1965, Gorodkov ( $10^{7}, 1$, ZISP); Tver' Oblast: Rzhev env. $\left(56.2^{\circ} \mathrm{N} 34.3^{\circ} \mathrm{E}\right), 18 . \mathrm{VIII} .2014, \mathrm{~N}$. Vikhrev $\left(10^{7}\right.$, ZMUM); Tyumen' Oblast: $\left(63.81^{\circ} \mathrm{N} 59.56^{\circ} \mathrm{E}\right), 6-8 . \mathrm{VII} .2010$, K. Tomkovich (1 $\mathrm{O}^{7}$, ZMUM); Labytnangi $\left(66.661^{\circ} \mathrm{N} 66.394^{\circ} \mathrm{E}\right)$, 5.VII.1974, Sychevskaya ( 1 ․, ZISP); Neroyka (ca. $\left.64.5^{\circ} \mathrm{N} 59.6^{\circ} \mathrm{E}\right), 9$ 21.VII. and 3-6.VIII.1990, Malozemov (3 $\sigma^{7} \sigma^{7}, 5$ 우, ZISP); Salekhard $\left(66.533^{\circ} \mathrm{N} 66.601^{\circ} \mathrm{E}\right), 17$.VII.1981, coll. XXX [XXX = illegible] (1 9 , ZMUM); Sob' station $\left(67.056^{\circ} \mathrm{N} 65.5104^{\circ} \mathrm{E}\right), 10$.VIII.1985, Gorodkov (1 $0^{7}$, ZISP); Yar near Tyumen' $\left(57.16^{\circ} \mathrm{N} 65.706^{\circ} \mathrm{E}\right)$, 5.IX.1976, Gorodkov (2 $\mathrm{O}^{7} \mathrm{O}^{7}$, ZISP); Repolovo $\left(60.658^{\circ} \mathrm{N} 69.822^{\circ} \mathrm{E}\right)$, 17 and 20.VI.1965, Veselkin (2 O+, ZMUM); Vorohezh Oblast: Voronezh env., Shilovskiy forest $\left(51.60^{\circ} \mathrm{N} 39.181^{\circ} \mathrm{E}\right)$, 7.IX.1965, Negrobov $\left(1 \sigma^{\circ}\right.$, ZISP); Usmanskiy forest $\left(51.911^{\circ} \mathrm{N} 39.562^{\circ} \mathrm{E}\right)$, 18.VIII.1970, Negrobov (1 O , 1 \% , ZISP);Vorohezh: Botanical Garden 
$\left(51.711^{\circ} \mathrm{N} 39.207^{\circ} \mathrm{E}\right), 15 . \mathrm{V} .1969$, Dorovskaya (1 $\sigma^{7}$, ZISP); Yaroslavl' Oblast: Berditsyno $\left(57.455^{\circ} \mathrm{N} 40.107^{\circ} \mathrm{E}\right)$, 20.VII.1907, A. Yakovlev (1 $\sigma^{\top}$, ZISP); Yaroslavl': (57.632 $\left.{ }^{\circ} \mathrm{N} 39.836^{\circ} \mathrm{E}\right), 2$.VI.1895, Kokuev (1 $\sigma^{\top}$, ZISP); Zabaikalsky Krai: Nerchinsk $\left(51.980^{\circ} \mathrm{N} 116.584^{\circ} \mathrm{E}\right)$, 1912, Faybushevich (1 О', ZISP).

ADDITIONAL MATERIAL. Kazakhstan: Katarkol', 18 km SO of Borovoe Lake $\left(52.946^{\circ} \mathrm{N} 70.431^{\circ} \mathrm{E}\right), 7$ and 11.VIII.1937, L. Zimin ( $1 \Im^{7}, 1$ ㅇ, ZISP).

DESCRIPTION. Male (Fig. 1), female. Mediumsized flies (5.0-7.8 $\mathrm{mm}$ long).

Head. Frontal and fronto-orbital plate yellow. Postcranium yellow completely or blackish in upper half. 3-4 frontal setae present. Antenna yellow. Postpedicel approximately 2 times as long as wide. Arista blackish, moderate haired: the length of hairs more than the width of basal diameter of arista.

Thorax yellow in ground color, delicately grey dusted. Scutum usually yellow with two blackish stripes along dorsocentral lines, rarely blackish completely between dorsocentral lines. Scutum with the following paired setae: $2+3$ dorsocentrals, a pair of scapular setae (usually small), 1+2 supra-alars, 1 postpronotal, 2 notopleurals, 2 postalars. Anepisternum with 2-3 setae along posterior margin. Scutellum as in Fig. 3, yellow or brown, grey dusted.

Legs entirely yellow, sometimes apex of mid and hind femora blackish. Fore femur with 6-10 long posteroventral and 6-9 short anteroventral setae. Fore tibia with 4 strong posteroventral and 3-6 strong anteroventral setae (Fig. 2). Mid tibia with 1 anteroventral, 1 anterodorsal, $1-2$ posterodorsal, $2-3$ posteroventral and a ring of apical setae, in male additionally with pale hairs ventrally. Hind tibia with 2-3 anterodorsal, 2 posterodorsal, 1 preapical dorsal and 1 apical anteroventral setae.

Wing tinged with brownish. Calypters, margins of calypters, and halteres yellowish.

Abdomen brown or blackish, greyish dusted. Female segments 7-8 shining. Male sternite 4 rectangular, approximately as wide as long (Fig. 5). Male sternite 5 with narrow curved internally lateral lobes (Fig. 17). Epandrium, cercal plate and surstyli as in Figs 46-48.

DISTRIBUTION. Russia: Adygea, Altai, Amur Oblast, Arkhangelsk Oblast, Bashkiria, Buryatia, Chelyabinsk Oblast, Chuvashia, Crimea, Dagestan, Irkutsk Oblast, Kaliningrad Oblast, Kamchatka, Karelia, Kemerovo Oblast, Khabarovsk Krai, Komi, Krasnodar Krai, Krasnoyarsk Krai, Kursk Oblast, Leningrad Oblast, Lipetsk Oblast, Magadan Oblast, Mari El Republic, Moscow and Moscow Oblast, Murmansk Oblast, Nizhegorod Oblast, North Ossetia - Alania, Novgorod Oblast, Novosibirsk Oblast, Omsk Oblast, Orenburg Oblast, Penza Oblast, Primorsky Krai, Pskov Oblast, Ryazan’ Oblast, Smolensk Oblast, Tatarstan, Tula Oblast, Tuva, Tver' Oblast, Tyumen' Oblast, Vorohezh Oblast, Yaroslavl' Oblast, Zabaikalsky Krai. — Widespread in Europe [Šifner, 2008], Iran [Khaghaninia \& Gharajedaghi, 2014], Kazakhstan (first record), Turkey [Eren, Ozerov, 2017], North America [Vockeroth, 1965].

\section{Norellisoma tomkovichi Ozerov, 2010}

Figs 18, 49-51.

tomkovichi Ozerov, 2010b: 158, 164 (Norellisoma). Typelocality: N Lagonaki mt., $>1830 \mathrm{~m}$ asl, $44.050^{\circ} \mathrm{N} 40.018^{\circ} \mathrm{E}$ (Adygea, Russia)

MATERIAL. Krasnodar Krai: Krasnaya Polyana $\left(43.679^{\circ} \mathrm{N}\right.$ 40.213 $\left.{ }^{\circ} \mathrm{E}\right), 25$.VI.2011, N. Vikhrev (1 $\sigma^{7}$, ZMUM); North Ossetia Alania: Zaramag $\left(42.696^{\circ} \mathrm{N} 43.999^{\circ} \mathrm{E}\right), 1900 \mathrm{~m}, 16 .-18 . V I I .1988$, A.L. Ozerov (1 + , ZMUM).

DESCRIPTION. Male, female. Medium-sized flies (4.3-5.2 mm long).

Head. Frontal vitta yellow; fronto-orbital plate yellow in lower half and blackish in upper half. Postcranium blackish in upper part and whitish in lower third. 2 frontal setae present. Antenna yellow. Postpedicel approximately 2 times as long as wide. Arista yellow, moderately haired: the length of hairs more than the width of basal diameter of arista.

Thorax and scutellum black, grey dusted. Scutum with the following paired setae: $1+2$ dorsocentrals, $1+2$ supra-alars, $0-1$ postpronotal, 2 notopleurals, 2 postalars. Anepisternum with 1 seta near posterior margin.

Legs entirely yellow (coxae of mid and hind legs yellow). Fore femur with 8-9 long posteroventral, 6-9 short anteroventral, 6 dorsal/posterodorsal setae. Fore tibia with 4 strong posteroventral and 5-6 strong anteroventral setae. Mid tibia with 1 anterodorsal, 1 posterodorsal and a ring of apical setae in both sexes, in male additionally with long pale hairs ventrally, in female with 1 anteroventral and 1 posteroventral setae. Hind tibia with 1-2 anterodorsal, 1-2 posterodorsal, 1 preapical dorsal and 1 apical anteroventral setae.

Wing tinged with yellowish. Calypters, margins of calypters, and halteres yellowish.

Abdomen black, greyish dusted, but tergites shining laterally. Female segments $7-8$ shining. Male sternite 4 approximately as wide as long. Male sternite 5 with long narrow parallel lateral lobes (Fig. 18). Epandrium, cercal plate and surstyli as in Figs 49-51.

DISTRIBUTION. Russia: Adygea, Krasnodar Krai, North Ossetia - Alania.

Norellisoma yolduense (Ozerov, 2008) Figs 19, 52-54.

yolduensis Ozerov, 2008: 3 (Norellia). Type-locality: $45 \mathrm{~km} \mathrm{E}$ of Ust'-Ulagan $\left(50.5^{\circ} \mathrm{N}, 88.57^{\circ} \mathrm{E}\right)$ (Altai, Russia).

NOTE. This species was known only from the typelocality.

MATERIAL. Altai: Kosh-Agach, Kurayskiy ridge, 2500-2700

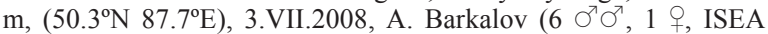
and ZMUM).

DESCRIPTION. Male, female. Medium-sized flies (6.4-6.8 mm long).

Head. Frontal vitta yellow; fronto-orbital plate yellow. Postcranium usually blackish in upper half and yellow in lower part, rarely yellow almost completely. 3-4 frontal setae present. Antenna yellow. Postpedicel approximately 2 times as long as wide. Arista blackish, short haired: the length of hairs about the width of basal diameter of arista. 
Thorax and scutellum from yellow to blackish, delicately grey dusted. Scutum often yellow with two blackish stripes along dorsocentral lines or blackish completely between dorsocentral lines. Scutum with the following paired setae: $2+3$ dorsocentrals, a pair of scapular setae (usually small), 1+2 supra-alars, 1 postpronotal, 2 notopleurals (posterior seta small), 2 postalars. Anepisternum with 2-3 setae near posterior margin.

Legs yellow, but usually mid and hind femora blackish dorsally near apex. Fore femur with 8-9 long posteroventral and 6-9 short anteroventral setae. Fore tibia with 4 strong posteroventral and 5-6 strong anteroventral setae. Mid tibia with 1 anteroventral, 1 anterodorsal, 1-2 posterodorsal, 2-3 posteroventral and a ring of apical setae. Hind tibia with $2-3$ anterodorsal, 2-3 posterodorsal, 1 anteroventral, 1 preapical dorsal and 1 apical anteroventral setae.

Wing tinged with brownish. Calypters, margins of calypters, and halteres brownish.

Abdomen black, greyish dusted. Female segments 7-8 shining. Male sternite 4 approximately as wide as long. Male sternite 5 with broad triangular lateral lobes (Fig. 19). Epandrium, cercal plate and surstyli as in Figs 52-54.

DISTRIBUTION. Russia: Altai.

Key to the SPecies of Norellisoma Wahlgren of Russia

1. Scutum with one pair of postsutural dorsocentral setae. Scutum black with yellow stripe between dorsocentral setae. Male sternite 5, epandrium, cercal plate and surstyli as in Figs 10, 26, 27 .......... . flavostriatum Ozerov

- Scutum with 2 or 3 pairs of postsutural dorsocentral setae......

2. Scutum with 2 pairs of postsutural dorsocentral setae …. 3

- Scutum with 3 pairs of postsutural dorsocentral setae .... 7

3. Fore coxa black. Femora of all legs completely black. Male sternite 5, epandrium, cercal plate and surstyli as in Figs 14, 37-39 ......... N. montanopratense (Ozerov)

- Fore coxa yellow. Femora of all legs yellow completely or darkened dorsally only .....

4. Postcranium black completely. Male sternite 5, epandrium, cercal plate and surstyli as in Figs 9, 23-25

\section{N. caucasicum (Ozerov)}

- Postcranium yellow in lower third or half

5. Fore tibia with 5 strong posteroventral setae. Male sternite 5, epandrium, cercal plate and surstyli as in Figs 13, 34-36 ....................................... N. lituratum (Meigén)

- Fore tibia with 4 strong posteroventral setae ................. 6

6. Thorax and scutellum black, densely grey dusted. Abdominal tergites 1-6 densely grey dusted. Height of gena below eye more than the width of postpedicel. Female sternite 6 with strong black setae near posterior margin. Male sternite 5, epandrium, cercal plate and surstyli as in Figs 12, 31-33....

N. lesgiae Becker

- Thorax and scutellum delicately grey dusted. Abdominal tergites 1-6 shining laterally. Height of gena below eye less than the width of postpedicel. Female sternite 6 with thin setae near posterior margin. Male sternite 5, epandrium, cercal plate and surstyli as in Figs 18, 49-51 ....

N. tomkovichi Ozerov

7. Male sternite 5 with a pair of short median projections on posterior margin (Figs 11, 16)
- Male sternite 5 without median projections on posterior margin (Figs 8, 19) ..................................................... 9

8. Length of median projection of male sternite 5 less than the distance between median projection and lateral lobe (Fig. 11). Epandrium, cercal plate and surstyli as in Figs 28-30. N. insulare (Ozerov)

- Length of median projection of male sternite 5 more than the distance between median projection and lateral lobe (Fig. 16). Epandrium, cercal plate and surstyli as in Figs 43-45. N. orientale (Ozerov)

9. Male sternite 5 with short lateral lobes (Fig. 8). Epandrium, cercal plate and surstyli as in Figs 20-22.

N. armipes (Meigén)

- Male sternite 5 with long lateral lobes (Fig. 19). Epandrium, cercal plate and surstyli as in Figs $52-54$

N. yolduense (Ozerov)

Acknowledgements. We are very grateful to Dr. Olga Ovchinnikova (ZISP) and Mrs. Galina Suleymanova (ZISP) for the loan of the material of Scathophagidae for study. The investigation was fulfilled within the state project No AAAAA16-116021660077-3 (A.L. Ozerov).

\section{References}

Bagachanova A.K., Ovchinnikov A.N., Ozerov A.L. 2016. On the Fauna of Scathophagidae (Diptera) of Yakutia // Entomological Review. Vol.96. No.6. P.775-785.

Becker T. 1894. Dipterologische Studien. I. Scatomyzidae // Berliner Entomologische Zeitschrift. Bd.39. H.1. S.77-196.

Cumming J.M., Wood D.M. 2009. Adult morphology and terminology // Brown et al. (eds.). Manual of Central American Diptera. Vol.1. National Research Council Press, Ottawa. P.9-50.

Disney R.H.L. 1976. The pre-adult stages of Norellisoma spinimanum (Fallén) (Dipt., Cordyluridae) and a parasitoid (Hym., Pteromalidae) of the same // Entomologist's Gazette. No.27. P.263-267.

Eren G., Ozerov A.L. 2017. The first record of Norellisoma spinimanum (Fallén, 1819) (Diptera: Scathophagidae) for the fauna of Turkey // Acta Biologica Turcica. Vol.30. No.2. P.61-63.

Fabricius J.C. 1794. Entomologia systematica emendata et aucta. Secundum classes, ordines, genera, species, adjectis synonimis, locis, observationibus, descriptionibus. Tom. IV. Hafniae: C.G. Proft, Fil. et Soc. 434 pp.

Fallén C.F. 1819. Scatomyzides. Lundae. 10 pp.

Ferrar P. 1987. A guide to the breeding habits and immature stages of Diptera Cyclorrhapha // Entomonograph. Vol.8. Part.1-2. $907 \mathrm{pp}$.

Gorodkov K.B. 1970. [Family Scathophagidae (Cordyluridae, Scatomyzidae, Scopeumatidae)] // Bei-Bienko G.Ya. (ed.). Key to the insects of the European part of the USSR. Vol.5. Diptera, Siphonaptera. Part 2. Leningrad. P.440-458 [in Russian].

Gorodkov K.B. 1986. Family Scathophagidae // Soós Á., Papp L. (eds.). Catalogue of Palaearctic Diptera. Vol.11. Scathophagidae-Hypodermatidae. Budapest: Akadémiai Kiadó. P.1141.

Hendel F. 1910. Über die Nomenklatur der Acalyptratengattungen nach Th. Beckers Katalog der paläarktischen Dipteren, Bd.4. // Wiener Entomologische Zeitung. Jg.29. S.307-313.

Hendel F. 1930. Entomologische Ergebnisse der schwedischen Kamtchatka-Expedition 1920-1922. 28. Diptera Brachycera 2. Fam. Cordyluridae und Dryomyzidae // Arkiv för Zoologi. Bd.21A. Nr.18. S.1-12.

Hironaga T., Suwa M. 2005. Notes on the genus Norellisoma in Japan, with description of a new species (Diptera, Scathophagidae) // Studia dipterologica. Vol.12. No.1. P.199-208.

Khaghaninia S., Gharajedaghi Y. 2014. Dung flies fauna (Diptera: Scathophagidae) in East Azerbaijan province of Iran, with 
eight new records // Journal of Crop Protection. Vol.3. No.3. P.389-396.

McAlpine J.F. 1981. Morphology and terminology-adults // McAlpine J.F., Peterson B.V., Shewell G.E., Teskey H.J., Vockeroth J.R., Wood D.M. (Coordinators). Manual of Nearctic Diptera. Vol.2. Ottawa: Research Branch. Agriculture Canada. Monograph 27. P.9-63.

Meigen J.W. 1826. Systematische Beschreibung der bekannten europäischen zweiflügeligen Insecten. Fünfter Theil. [5]. Hamm. $412 \mathrm{~S}$.

Ovchinnikov A.N. 2013. [Dvukrylye semeistva Scathophagidae (Diptera) evropeiskoy chasti Rossii: vidovoi sostav, rasprostranenie i morfoadaptivnye preobrazovania yaitseklada]. $\mathrm{PhD}$ Thesis. St-Petersburg: Zoologicheskiy Institut RAN. 151 pp. [In Russian]

Ozerov A. L. 1993. New palaearctic species of the genus Norellia (Diptera, Scathophagidae // Vestnik Zoologii. No.5. P.76-93 [in Russian].

Ozerov A.L. 2008. Two new species of the genus Norellia Robineau-Desvoidy, 1830 (Diptera, Scathophagidae) from Altai // Far Eastern Entomologist. No.184. P.1-4.

Ozerov A.L. 2009. New species of Scathophagidae (Diptera) // Russian Entomological Journal. Vol.17 (for 2008). No.4. P.419427.

Ozerov A.L. 2010a. On the Palaearctic fauna of Norellisoma Wahlgren, 1917 (Diptera: Scathophagidae) // Russian Entomological Journal. Vol.18 (for 2009). No.3. P.229-234.

Ozerov A.L. 2010b. Five new species of Scathophagidae (Diptera) from Russia // Russian Entomological Journal. Vol.19. No.2. P.157-166.
Ozerov A.L., Krivosheina M.G. 2011. The first record of Norellia tipularia (Fabricius, 1794) (Diptera, Scathophagidae) for the fauna of Russia // Russian Entomological Journal. Vol.20. No.1. P.101-103.

Ozerov A.L., Krivosheina M.G. 2014. To the fauna of dung flies (Diptera: Scathophagidae) of Russian Far East // Russian Entomological Journal. Vol.23. No.3. P.203-222.

Rondani C. 1866. Scatophaginae Italicae collectae distinctae et in ordinem dispositae. Dipterolodiae Italicae Prodromus. Pars.VII. Fasc.I. Milano: Bernardoni. 52 pp.

Roser C., von. 1840. III. Beiträge zur Vaterlandskunde. Erster Nachtrag zu dem in Jahre 1834 bekannt gemachten Verzeichnisse in Württemberg vorkommender zweiflügliger Insekten // Correspondenzblatt des Koniglich Württembergischen Landwirtschaftlichen Vereins. Bd.1. H.1. S.49-64.

Šifner F. 2008. A catalogue of the Scathophagidae (Diptera) of the Palaearctic region, with notes on their taxonomy and faunistics // Acta Entomologica Musei Nationalis Pragae. Vol.48. No.1. P.111-196.

Stuckenberg B.R. 1999. Antennal evolution in the Brachycera (Diptera), with a reassessment of terminology relating to the flagellum // Studia Dipterologica. Vol.6. S.33-48.

Vockeroth J.R. 1965. Subfamily Scatophaginae // Stone A. et al. (eds.). A catalog of the Diptera of America north of Me-xico. United States Department of Agriculture. Agriculture Handbook. No.276. P.826-842.

Wahlgren E. 1917. 6. Fam. Kolflugor. Cordyluridae // Svensk Insectfauna, Entomologiska Foreiningen, Stockholm. P.132160.

Zetterstedt J.W. [1838]. Sectio tertia. Diptera // Insecta Lapponica.'1840'. Lipsiae [= Leipzig]: Leopold Voss. P. 477-868 